\title{
Maximum expected ramp rates using cloud speed sensor measurements
}

Cite as: J. Renewable Sustainable Energy 12, 056302 (2020); https://doi.org/10.1063/5.0021875 Submitted: 15 July 2020 . Accepted: 13 August 2020 . Published Online: 25 September 2020

Guang Chao Wang (王光超), Ben Kurtz, Juan Luis Bosch, íñigo de la Parra, and (D) Jan KleissI
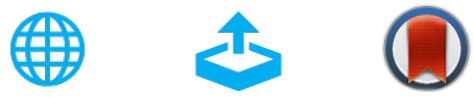

View Online

\section{ARTICLES YOU MAY BE INTERESTED IN}

Response of lipid productivity to photosynthesis of Chlorella vulgaris under various nutrient stress modes

Journal of Renewable and Sustainable Energy 12, 056102 (2020); https://

doi.org/10.1063/1.5144539

Quantifying the spatial scale mismatch between satellite-derived solar irradiance and in situ measurements: A case study using CERES synoptic surface shortwave flux and the Oklahoma Mesonet

Journal of Renewable and Sustainable Energy 12, 056104 (2020); https://

doi.org/10.1063/5.0025771

Temperature impacts on utility-scale solar photovoltaic and wind power generation output over Australia under RCP 8.5

Journal of Renewable and Sustainable Energy 12, 046501 (2020); https://

doi.org/10.1063/5.0012711 


\title{
Maximum expected ramp rates using cloud speed sensor measurements
}

\author{
Cite as: J. Renewable Sustainable Energy 12, 056302 (2020); doi: 10.1063/5.0021875 \\ Submitted: 15 July 2020 - Accepted: 13 August 2020 . \\ Published Online: 25 September 2020

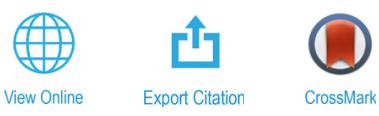

\author{
Guang Chao Wang (王光超), (D) Ben Kurtz, (iD) Juan Luis Bosch, ${ }^{2}$ ínigo de la Parra, ${ }^{3}$ and Jan Kleissl ${ }^{1}$ (iD
}

\author{
AFFILIATIONS \\ ${ }^{7}$ Center for Renewable Resource Integration, Department of Mechanical and Aerospace Engineering, University of California, \\ San Diego, CA, USA \\ ${ }^{2}$ Department of Chemistry and Physics, University of Almera, 04120 Almera, Spain \\ ${ }^{3}$ Institute of Smart Cities (ISC), Department of Electrical, Electronic and Communications Engineering, Public University of Navarre, \\ Arrosadia Campus, 31006 Pamplona, Spain
}

\begin{abstract}
Large ramps and ramp rates in photovoltaic (PV) power output are of concern and sometimes even explicitly restricted by grid operators. Battery energy storage systems can smooth the power output and maintain ramp rates within permissible limits. To enable PV plant and energy storage system design and planning, a method to estimate the largest expected ramps for a given location is proposed. Because clouds are the dominant source of PV power output variability, an analytical relationship between the worst expected ramp rate, cloud motion vector, and the geometrical layout of the PV plant is developed. The ability of the proposed method to bracket actual ramp rates is assessed over 10 months under different meteorological conditions, demonstrating an average compliance rate of $98.9 \%$ for a 2 min evaluation time window. The largest observed ramp of $29.7 \% \mathrm{~s}^{-1}$ is contained with the worst case estimate of $34.3 \% \mathrm{~s}^{-1}$. This method provides a convenient yet economical approach to worst-case PV ramp rate modeling and is compatible with solar irradiance measured at coarse temporal resolution.
\end{abstract}

Published under license by AIP Publishing. https://doi.org/10.1063/5.0021875

\section{INTRODUCTION}

The power output variability by large-scale grid-connected photovoltaic (PV) systems (https://www.sciencedirect.com/topics/materialsscience/photovoltaics) can negatively affect power quality and grid network reliability. Regulations have been introduced to restrict the maximum power ramp rates for PV plants on 1 min timescales (Gevorgian and Booth, 2013). These restrictions typically invite one of two approaches: (1) Compensate the power variability through energy storage systems (ESSs). The storage requirements and strategies to comply with the regulations, considering capacity losses and cycling degradations, have been comprehensively studied (Ru et al., 2014; de la Parra et al., 2015; Marcos et al., 2014a). (2) Curtail the PV output to smooth up-ramps reactively and provide a buffer for smoothing down-ramps proactively (Tonkoski et al., 2011; Shivashankar et al., 2016). For example, short-term forecasts for future cloud arrivals allow a system operator to meet ramp rate restrictions with less battery reserve or curtailment (Nguyen et al., 2016; Kuhn et al., 2018a; Saleh et al., 2018). If all ramp rates (except plant outages) were to be mitigated, approach (1) would require knowledge of the worst-case ramp rate to determine the power and energy rating of the ESS. Given perfect forecasts, approach (2) could mitigate all power ramps without ESSs. In practice, however, significant errors in the short-term ramp forecasts combined with restrictive ramp rate compliance requirements typically still require an ESS to mitigate worst-case ramp rates, but accurate forecasts can reduce the number of charge/discharge cycles of the ESS.

Although the nature of PV power variability has been wellstudied (Perez et al., 2010; Hoff and Perez, 2010; Lave et al., 2012; Lave et al., 2015; Perez et al., 2016), existing approaches to simulate power fluctuations are expensive or cumbersome. For example, Kuszamaul et al. (2010) created a network with synchronized sensors throughout the plant and approximated power output fluctuations as the average of the sensor readings. Marcos et al. (2011) studied the smoothing effect of power fluctuations over the area of the power plant by lowpass filtering irradiance measurements at a single point, but the model needs to be empirically tweaked for each PV plant. Lave et al. (2013) proposed a wavelet variability model to simulate the reduction in power output fluctuations of a plant or a fleet of dispersed plants. The correlation scaling coefficient introduced in the model is universal and a function of cloud speed (Lave and Kleissl, 2013). Marcos et al. (2016) simulated the power output by a fleet of plants, using only irradiance measurements at a single location and the smoothing effect due to geographical dispersion and plant size. 
To the authors' knowledge, all solar power high frequency variability models in the literature require high frequency $[\mathrm{O}(1 \mathrm{~s})]$ solar irradiance measurements (Van Haaren et al., 2015; de la Parra et al., 2018). The sensitivity of solar variability model performance influenced by low frequency solar irradiance data is demonstrated in Lave et al. (2015). One-minute resolution data were found to be barely acceptable for ramp simulations with the induced errors being within the margin of other modeling errors. While high frequency solar irradiance data are rarely available, some applications, such as PV plant design and ESS sizing, require only the worst power fluctuations. For example, the worst ramp rate determines the required power and energy capacity rating for the ESS to buffer all down-ramps (Marcos et al., 2014b). Motivated by this, we proposed a novel analytical approach, where the maximum expected PV ramp rate is computed in a process-based model using (1) low-resolution point irradiance or PV power measurements, (2) the geometrical layout of the PV plant, and (3) cloud velocity. During a cloud passage, the power ramp amplitude depends on the cloud optical depth, and the ramp rate is a function of both the optical depth and how fast a moving cloud passes over the plant. We utilize cloud motion vectors (CMVs) at the ground level measured by our unique and recently upgraded cloud speed sensor (CSS) (Fung et al., 2014; Wang et al., 2016; Kuhn et al., 2018b).

In this paper, we advance our previous work (Wang et al., 2019; Lappalainen et al. 2020) through a more thorough derivation and demonstration of a simple analytical model that bounds the maximum ramp rate with greater accuracy, and we evaluate the capability of the model over 90 days, spanning all seasons and multiple cloud types. As CMVs are a key input of the model, we also take this opportunity to fully test and validate the performance of the improved version of the CSS, which measures CMVs more accurately in real-time and at a higher sampling rate.

The remainder of this paper is organized as follows. Sections II A and II B present the ramp rate model as an analytical relation between cloud velocity, PV plant dimension, recent PV power/cloud optical depth measurements, and expected maximum ramp rate. Section II C introduces metrics to evaluate the model. Sections III A and III B clarify the motivation for the specific validation approach adopted in this paper and describe the experimental setup and data in detail. Section III C introduces recent CSS advancements in hardware design and real-time data processing, and Section IIID introduces the process to derive the data input for the model. Sections IV A and IV B examine the model performance through detailed analysis for an example day and validations over a 10 -month period. Sections IV C-IV G discuss the performance, application, and benefits of the model in detail. Finally, Section V provides the conclusions.

\section{MATHEMATICAL DERIVATION AND PROBLEM FORMULATION}

\section{A. A Geometric ramp rate model}

The geometric ramp rate model is based on the following three assumptions: (1) Advection of a large frozen cloud: A known irradiance field moving at a constant cloud motion vector impacts a PV plant. The irradiance field is larger than the size of the PV plant. The irradiance field contains a homogenous cloud layer with a stationary optical thickness over the time period when the ramp occurs. (2) No mismatch losses in the PV plant. Thus, the PV plant power is proportional to spatially averaged irradiance. In reality, PV system efficiency in partly cloudy conditions would be expected to be reduced due to partial shading and mismatch between the output of different cells on a string. (3) Constant PV efficiency, i.e., no cell temperature or inverter effects. Assumptions (2) and (3) simplify the irradiance-to-power model.

With these assumptions, we start with a simple $1 \mathrm{D}$ example where a power ramp results solely from an irradiance field moving eastward over a PV plant of size $S$, as conceptualized in Fig. 1(a). For simplicity, but without loss of generality, the irradiance field represented in Fig. 1 consists of a cloud (bordered by the gray rectangle) and clear sky elsewhere (white space). The ramp rate is modeled based on the interaction between this irradiance field advected by the CMV and the PV plant geometry, which, in our model, is aligned with North-South directions. At the initial time $t_{0}$, the cloud (solid border)

$$
t=t_{0}+\Delta t
$$

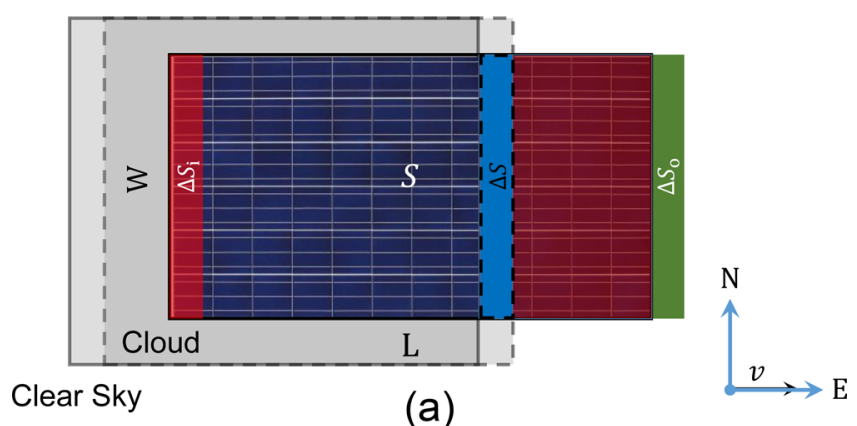

Clear Sky

(a)

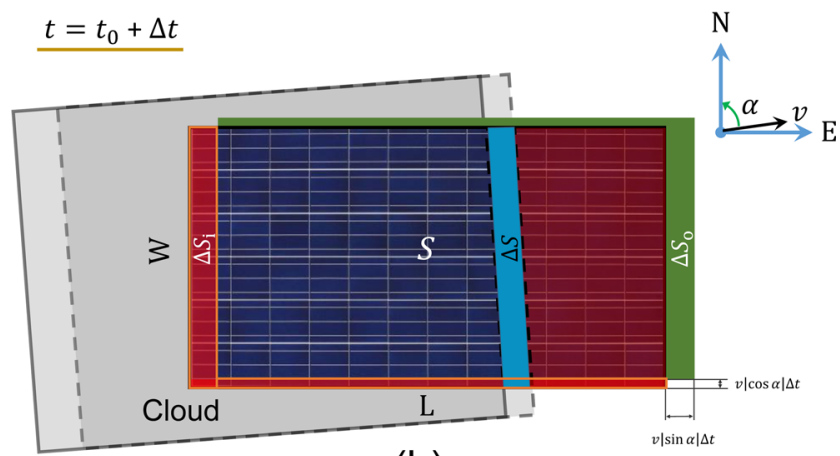

Clear Sky

(b)

FIG. 1. Conceptual illustration of modeling the geometric relation between the ramp rate and the movement of the irradiance field in $1 \mathrm{D}(\mathrm{a})$ and $2 \mathrm{D}(\mathrm{b})$ scenarios. The irradiance field is defined by a cloud (gray rectangle labeled "Cloud") embedded in clear sky (surrounding white spaces labeled "Clear Sky"). The PV plant measures $S=L$ by $W$ and, for convenience, aligns with North-South directions. The cloud intersecting the PV plant at $t_{0}$ (solid border) is being advected at speed $v$ and in direction $\alpha$ to a new position (dashed border). The incoming area $\Delta S_{\mathrm{i}}$ (red) defines the area of the plant that is covered by the region of the irradiance field previously outside of the plant. Conversely, the outgoing area $\Delta S_{0}$ (green) defines the portion of the irradiance field that previously covered the plant, but moves outside the plant during time interval $\Delta t$, with $\Delta S_{i}=\Delta S_{0}=\Delta S$ in (a) and $\Delta S_{i}=\Delta S_{0} \geq \Delta S$ in (b) per Appendix B. While the two complementary areas result in a power ramp event in a complex mathematical relation [Eq. (5)], the plant area that is effectively impacted by the cloud advection $\Delta S$ is painted in blue for illustration clarity. 
covers a part of the PV plant and then is advected during a small time interval $\Delta t$ into a new position (dashed border). During the advection, part of the irradiance field moves off the plant (i.e., the green outgoing area is denoted as $\Delta \mathrm{S}_{\mathrm{o}}$ ), while the complementary upwind part moves in (i.e., the red incoming area is denoted as $\Delta \mathrm{S}_{\mathrm{i}}$ ). $\Delta \mathrm{S}_{\mathrm{o}}$ and $\Delta \mathrm{S}_{\mathrm{i}}$ are of the same size. Note that it is the difference in the averaged cloud optical thickness between $\Delta S_{\mathrm{o}}$ and $\Delta S_{\mathrm{i}}$ that induces a power ramp event. An alternate, more intuitive presentation of the effectively affected area $\Delta S$ as a result of the movement of solar radiance field within $\Delta t$ is highlighted in blue. In this $1 \mathrm{D}$ example, $\Delta S=\Delta S_{\mathrm{i}}=\Delta S_{\mathrm{o}}=\mathrm{W} v \Delta t$.

A more general 2D example is conceptualized in Fig. 1(b). The cloud motion vector is not aligned with the orientation of the plant (i.e., cloud direction $\alpha \neq 90^{\circ}$ ), yielding a composite shape $\Delta \mathrm{S}_{\mathrm{i}}$ composed of a vertical rectangle and a horizontal rectangle (both in orange border), and their respective complementary areas $\Delta \mathrm{S}_{\mathrm{o}}$ as well. The total area of the composite shape $\Delta \mathrm{S}_{\mathrm{i}}$ and $\Delta \mathrm{S}_{\mathrm{o}}$ can be formularized as follows:

$$
\Delta \mathrm{S}_{\mathrm{o}}=\Delta \mathrm{S}_{\mathrm{i}}=(\mathrm{L} v|\cos \alpha|+\mathrm{W} v|\sin \alpha|) \Delta t-(v \Delta t)^{2}|\sin \alpha \cos \alpha| .
$$

For illustration clarity, the effectively affected area $\Delta S$ is again highlighted in blue, which is responsible for the power change and can be calculated as $\Delta S=\mathrm{W} v \Delta t /|\sin \alpha|$, as per the trigonometric diagram in Appendix B. Nevertheless, we use the full impacted area $\Delta S_{i}$ and $\Delta \mathrm{S}_{\mathrm{o}}$ as indicated in Eq. (1) to derive the analytical relation between the system geometry and the power ramp rate.

For reference, the area-normalized clear sky power production $\hat{P}_{\text {cs }}$ is calculated by Eq. (2), given the power production under clear sky condition $P_{\text {cs }}$,

$$
\hat{P}_{\mathrm{cs}}=\frac{P_{\mathrm{cs}}}{\mathrm{LW}} .
$$

The solar power that would be produced by $\Delta S_{\mathrm{i}}$ under a clear sky condition can be expressed as

$$
P^{*}=\hat{P}_{\mathrm{cs}} \Delta S_{\mathrm{i}}
$$

The solar power change $\Delta P$ caused by a change in the cloud optical thickness between $\Delta S_{\mathrm{i}}$ and $\Delta S_{\mathrm{o}}$ is described using the clear sky index $k_{\mathrm{cs}}$ (Ineichen and Perez, 2002) as

$$
\Delta P=\left(k_{\mathrm{cs}_{\mathrm{i}}}-k_{\mathrm{cs}_{\mathrm{o}}}\right) P^{*},
$$

where $k_{\mathrm{cs}_{\mathrm{i}}}$ and $k_{\mathrm{cs}_{0}}$ represent the average cloud optical thickness for $\Delta S_{\mathrm{i}}$ and $\Delta S_{\mathrm{o}}$ of the irradiance field, respectively. Since the irradiance field is a mix of cloudy and clear sky conditions, $k_{\mathrm{cs}_{\mathrm{i}}}-k_{\mathrm{Cs}_{\mathrm{o}}}$ can be positive or negative. Finally, the ramp rate RR becomes

$$
\begin{aligned}
\mathrm{RR} & =\frac{\Delta P}{\Delta t} \\
& =\frac{\left|k_{\mathrm{cs}_{\mathrm{i}}}-k_{\mathrm{cs}_{\mathrm{o}}}\right| P_{\mathrm{cs}}}{\Delta t \mathrm{LW}}\left((\mathrm{L} v|\cos \alpha|+\mathrm{W} v|\sin \alpha|) \Delta t-(v \Delta t)^{2}|\sin \alpha \cos \alpha|\right) .
\end{aligned}
$$

\section{B. The worst-case scenario ramp rate (WCS-RR)}

Equation (5) is not intended for operational ramp rate forecasts since-without a sky imager-the upwind irradiance field that would be needed to quantify $k_{\mathrm{csi}_{\mathrm{i}}}$ is generally not available. Instead, we consider the worst-case scenario, where a clear sky gives way to an overcast sky. We estimate the largest ramp rate by picking the largest and smallest $k_{\mathrm{cs}}$ from recent history (e.g., $\left.30 \mathrm{~min}\right) k_{\mathrm{cs}_{\max }}$ and $k_{\mathrm{cs}_{\min }}$. The WCS-RR can be expressed as

$$
\begin{aligned}
\mathrm{WCS}-\mathrm{RR}= & \pm \frac{\left|k_{\mathrm{cs}_{\max }}-k_{\mathrm{cs}_{\text {min }}}\right| P_{\mathrm{cs}}}{\Delta t \mathrm{LW}} \\
& \times\left((\mathrm{L} v|\cos \alpha|+\mathrm{W} v|\sin \alpha|) \Delta t-(v \Delta t)^{2}|\sin \alpha \cos \alpha|\right) .
\end{aligned}
$$

Note that $k_{\mathrm{cs}_{\max }}$ is not necessarily $k_{\mathrm{cs}}$ in the clear sky condition. For example, due to additional diffuse irradiance, cloud edge enhancement can cause irradiance to exceed the clear sky irradiance (Lave et al., 2012). Furthermore, we consider a specific time period of the cloud passage that warrants the worst-case scenario, which is not reflected in Eq. (5), but the reader is referred to Appendix $C$ for detailed discussions. Finally, Eq. (6) presents an analytical estimate of the expected maximum ramp rate given cloud velocity, largest and smallest $k_{\mathrm{cs}}$, and PV plant dimension.

\section{Performance evaluation}

The WCS-RR estimate is evaluated by the following performance metrics. First, we define the compliance indicator $\sigma$ by dividing the actual ramp rate by the corresponding WCS-RR estimate, as in the following equation:

$$
\sigma(t)=\frac{\mathrm{RR}^{\text {actual }}(t)}{\operatorname{RR}^{\text {estimate }}(t)} .
$$

When $\sigma \leq 1$, the actual ramp rate complies with the WCS-RR estimate. The maximum $\sigma$ in each non-overlapping evaluation window of length $m$ minutes is

$$
\mu_{j}=\max \{\sigma(i) ; i \in[n j, n(j+1)-1]\}, \quad j \in[1, N] .
$$

$n=(m \times 60) \mathrm{s} / R$ represents the number of $\sigma$ in the $j$ th evaluation window with a temporal resolution of $R \mathrm{~s}$, and $N$ is the total number of evaluation windows computed by rounding up the expression $T / m$, in which $T$ is the overall daily time window of the WCS-RR in minutes. The selection of $m$ is somewhat arbitrary: a shorter window length results in more windows with exclusively clear or overcast conditions-which are not of concern for ramp rates-while longer window lengths tend to evaluate $\sigma$ too infrequently. Since transmission system operators are typically required to counteract power fluctuations with the load following at a time scale of less than $30 \mathrm{~min}$, we apply window lengths of $m=2,10$, and $30 \mathrm{~min}$.

The compliance rate $\phi$ is defined as

$$
\phi=\frac{N_{\text {cpl }}}{N} .
$$

The number of compliance events $N_{\text {cpl }}$ indicates the number of windows that satisfy $\mu_{j} \leq 1$. Subsequently, the noncompliance rate becomes

$$
\epsilon=(1-\phi) \times 100 \% .
$$

Note that $\epsilon$ characterizes the occurrence in percentage that the maximum actual ramp in the evaluation window does not comply with the corresponding WCS-RR estimate. To further clarify this concept, we 
present the way we compute it in Appendix E using one example day in our analysis.

While risk-adverse actors would prefer that the WCS-RR always envelopes the observed ramps, excessive WCS-RR may result in an over-sized energy storage system. To quantify the extent to which the WCS-RR overpredicts the actual ramp, all compliance events are further evaluated by the degree of overestimation $\delta$,

$$
\delta=\left(\frac{1}{N_{\mathrm{cpl}}} \sum_{j \in N_{\mathrm{cpl}}} 1-\mu_{j}\right) \times 100 \% .
$$

Note that the summation term in Eq. (11) is not a continuous series, but rather it includes only the compliance events where $\mu_{j} \leq 1$.

\section{VALIDATION SETUP AND DATA \\ A. Motivation for the chosen validation approach}

To validate the proposed method, we setup an experiment at the University of California San Diego (UCSD) campus test bed. The validation is going to be inconsistent with the stated objective of the method, and this paragraph clarifies and resolves these inconsistencies. The objective of the WCS-RR method is to size ESSs that can mitigate the worst-case situations to ensure a $100 \%$ compliance rate with ramp rate restrictions. To satisfy this objective, only the single worst ramp rate is needed, and time-resolved ramp rates are unnecessary. While the WCSRR method would only be validated with a single data point per site, we instead validate a time-series implementation of the method where the WCS-RR is compared within evaluation windows against observed worst ramp rates. While that is not necessary for the objective of the WCS-RR method, our time-resolved validation approach provides a larger validation sample size and allows relational analysis between the time-resolved physics variables, such as cloud speed and cloud optical depth, and the modeled and measured ramp rates.

A note on the time resolution of the input data is also in order. As advertised, the advantage of the method is that it can be applied to coarse resolution (e.g., hourly) input data. Such data are commonly available: for example, hourly output of the cloud optical thickness (or $\mathrm{GHI}$ ) and CMV from numerical weather prediction (NWP) or reanalysis could be input to Eq. (6) to create a time-series of the WCS-RR. Alternatively, hourly GHI from ground stations and CSS data or CMV from ground sensors (Bosch and Kleissl 2013; Bosch et al., 2013) could be used. However, higher resolution data will benefit the accuracy of the method as some short-lived cloud phenomena, such as irradiance enhancement or deep convection, may yield unreasonable ramp rates (larger cloud optical depth and/or larger cloud speed) with the hourly data resolution. Safety factors could be considered if hourly or coarser data are used. Finally, if very high resolution $[\mathrm{O}(1 \mathrm{~s})]$ data are available, then power variability models, such as those presented in the introduction, are recommended as they provide more accurate information on the worst-case ramp rates and their time-series ramp rate estimates can be directly applied to energy storage simulation models.

\section{B. PV Data}

Figure 2 illustrates the layout of two existing PV systems located on the EBU2 building $\left(32^{\circ} 52^{\prime} 53.1^{\prime \prime} \mathrm{N}, 117^{\circ} 13^{\prime} 59.2^{\prime \prime} \mathrm{W}\right)$ at UCSD with a tilt angle of $20^{\circ}$ and an azimuth angle of $225^{\circ}$ east of north. The system under study is arranged in three arrays, consisting of a total of 181 PV panels (marked in red in Fig. 2) with overall dimensions of $33.5 \mathrm{~m}$ $\times 16 \mathrm{~m}$. The total nominal power is $37.1 \mathrm{~kW}$ DC. The PV power was measured at a sampling rate of $2 \mathrm{~s}$ from September 29, 2017 to October 1, 2018 by 5 inverters, including two SMA Sunny Boy 5000US and three SMA Sunny Boy 7000US, with a total rated power of $31 \mathrm{~kW}$ AC. The PV database consists of 10 months of power output measurements. Excluding server shutdowns, rainy and overcast days, and clear days (defined as less than 30 min of cloud cover), 90 partially cloudy days remain. Only partial cloud cover is of interest to the experiment because it causes the largest power ramps. Note that the production field includes the ground area in-between the rows, which mathematically enters Eq. (2) through the power plant dimensions. To avoid errors from clear sky and PV performance models, the power produced on the most recent clear day is used as the clear sky power.

\section{Cloud speed sensor (CSS) and recent improvements}

The cloud speed and direction are required to relate the cloud field to ramp rates. While the proposed model is compatible with any type of cloud speed measurement, we obtain cloud speed measurements from our in-house CSS, which provides an accurate yet affordable means to measure local CMVs. Refer to Wang et al. (2016) for detailed design specifications and features. The instrument is installed on the same rooftop as the PV system and marked in Fig. 2.

The CSS consists of a set of nine phototransistors arranged about a semi-circle. In the original CSS design, $666 \mathrm{~Hz}$ raw data were measured in $18 \mathrm{~s}$ segments. For processing, data had to be sent to a remote server via an Ethernet card. In the upgraded CSS, the existing microcontroller chipKIT Max32 still collects raw data, but now transmits the data to an attached Raspberry Pi through a much faster serial connection. This Raspberry Pi directly computes the CMV based on the Linear Cloud Edge (LCE) assumption, using a $9 \mathrm{~s}$ set of 6000 samples from the phototransistors. The updated hardware design processes each $9 \mathrm{~s}$ batch of sampled data in only $2 \mathrm{~s}$, outputting the CMVs at $11 \mathrm{~s}$ resolution. This resolution is sufficient for cloud motion estimation in power variability modeling. Continuous sampling would be feasible with additional software upgrades. The final processed CMV measurements are stored onboard along with the raw sensor data and timestamps. To support the external real-time use of the data, CMV data are also published to the local network via the MODBUS TCP protocol. The upgraded CSS has been fully operational since October 8, 2017.

\section{Enhanced CSS software to extract real cloud velocity}

An enhanced CMV calculation algorithm addressing limitations of the LCE assumption is introduced in this subsection. The CSS algorithm in Wang et al. (2016) assumes a linear cloud edge passing over the array of sensors given the fact that clouds are typically much larger than the spacing between sensors $(0.078 \mathrm{~m})$ and, therefore, only detects the component of the velocity that is perpendicular to the cloud edge, which systematically underestimates the speed (vector magnitude) slightly. Figure 3 illustrates that under the LCE assumption, the CSS would report different CMVs from a single cloud. To resolve this ambiguity that was left for future work in Wang et al. (2016), we compute actual cloud velocity $|\boldsymbol{v}|$ using the reported perpendicular cloud speeds $v_{\perp}^{i}$ and directions $\phi_{\perp}^{i}$, following Eqs. (6) and (7) in Wang et al. (2016) through a weighted non-linear regression of $|v|$ and $\alpha$ to the $N$ collected CMVs in a recent period (Sec. III D) using 


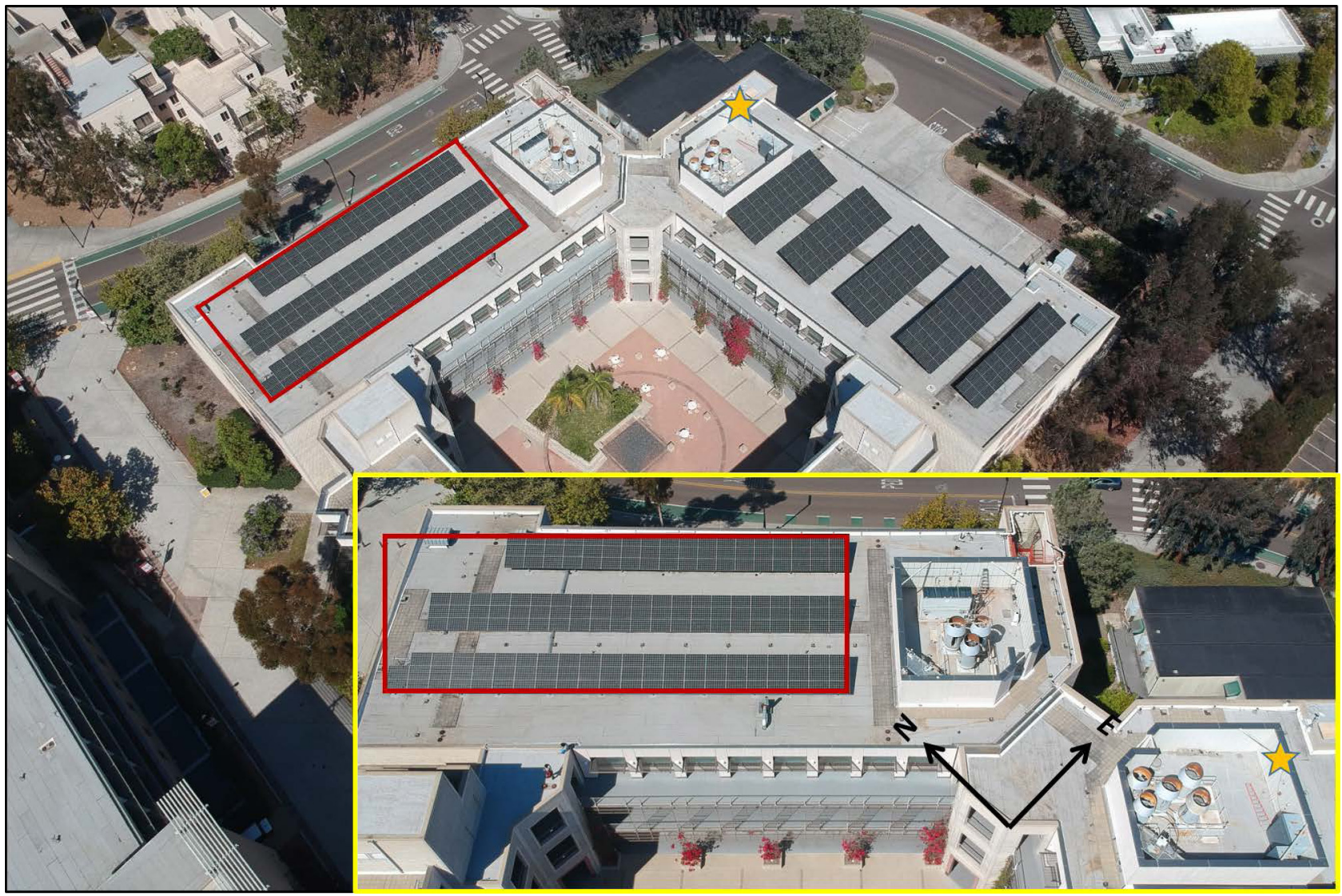

FIG. 2. Aerial view of the PV systems installed on EBU2 at UCSD. Plane view of the PV system (red rectangle) considered in the ramp estimate model is the inset at bottom right. The yellow star indicates the cloud speed sensor (see Sec. III C) located $30 \mathrm{~m}$ east of the lower right corner of the PV system. Copyright Drone Photo by Guang Chao Wang.

$$
\begin{aligned}
& w_{i}\left|v_{\perp}^{i}\right|=|\boldsymbol{v}| \cos \left(\phi_{\perp}^{i}-\alpha\right), \\
& \text { where } w_{i}=\frac{1}{t_{o}-t_{i}}, \forall i \in N .
\end{aligned}
$$

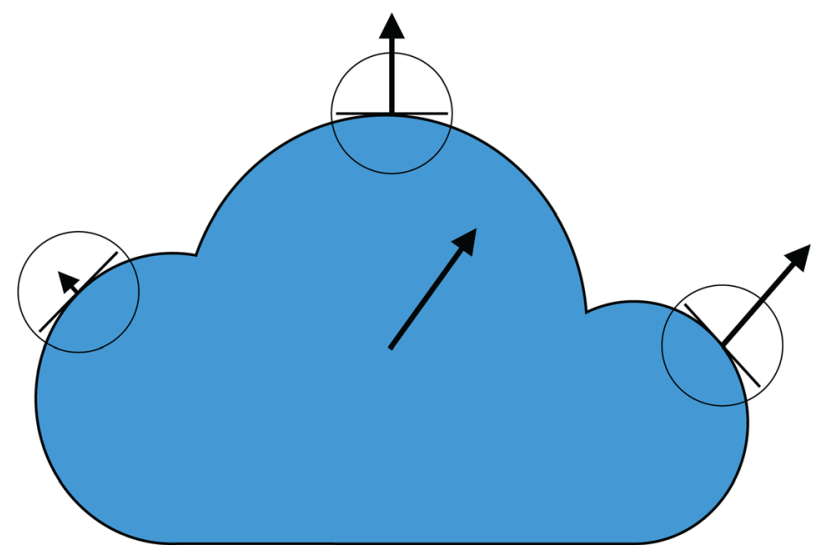

FIG. 3. Schematic depiction of actual cloud velocity (centered arrow) vs perpendicular components (other arrows) derived from different cloud edge orientations in the prior CSS algorithm in Wang et al. (2016). $|v|$ represents the magnitude of the vector of actual cloud velocity $\boldsymbol{v} . w_{i}$ represents the weighting factor computed as the inverse of the time difference between the end point of the time window (i.e., present time $t_{o}$ ) and the timestamp of the $i$ th $\mathrm{CMV}\left(t_{i}\right)$. As a result, the most recent CMV has the highest weight.

While $\boldsymbol{v}$ becomes unreliable when $\phi_{\perp}$ exhibits only small variations, small $\phi_{\perp}$ variation suggests only minor changes in the LCE orientation of the cloud field. Hence, if the collected $N$ raw CMVs differ by less than $20^{\circ}$, the reported CMV is reasonably considered as the true CMV and the regression in Eq. (12) is not conducted. Instead, the CMVs are decomposed into horizontal and vertical directions, and the median value of each is then used to reconstruct one median filtered CMV.

\section{Search time window and data processing}

Since CMVs are only available in irregular time intervals, a search window length needs to be defined to average the CMV data and select the largest and smallest $k_{\mathrm{cs}}$. Longer windows challenge the assumption of cloud field homogeneity, causing older cloud fields that are likely different from the ones at present to be counted. Shorter windows may not contain sufficient cloud cover events and falsely suggest that clear conditions will persist. Based on our experience, the cloud field 


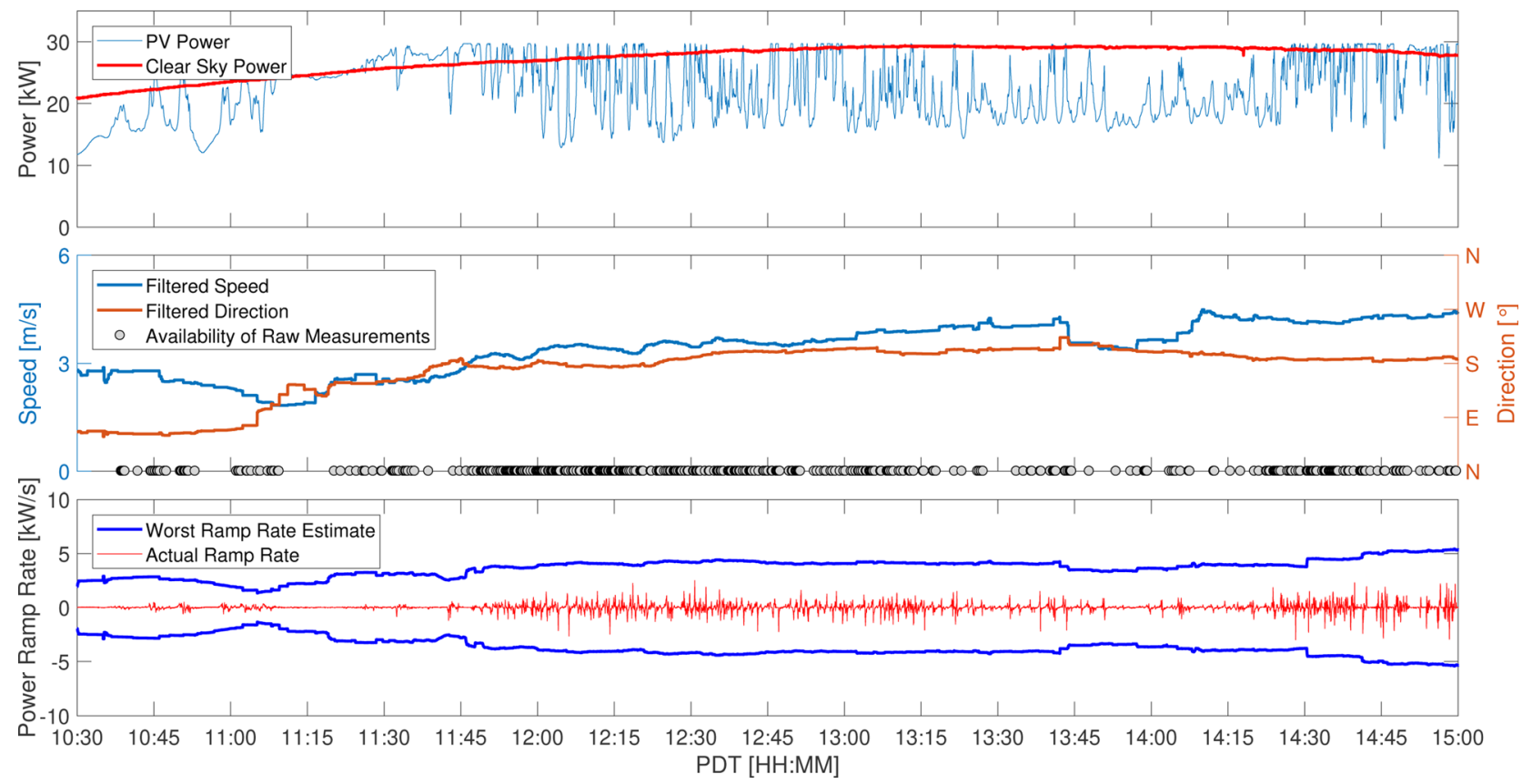

FIG. 4. Example validation of the proposed method on June 21, 2018. Top: actual PV power on June 21, 2018 (blue) and on the most recent clear day (June 19, 2018, red). Middle: the true velocity and directions (lines) are derived from non-linear regression [Eq. (12)] on the raw CSS CMV measurements (their availabilities are indicated by dots) collected in the centered 30 min time window. Bottom: comparison between the actual ramp rate and the WCS-RR estimates.

in coastal Southern California is typically steady over a couple of hours, and so we consider a $30 \mathrm{~min}$ window centered at the time of interest as a conservative upper bound. For areas with a faster changing cloud pattern and more short-lived cloud phenomena, a smaller time window would be preferred.

The search window is also needed to determine appropriate $k_{\mathrm{cs}}$ values in Eq. (6). $k_{\mathrm{cs}}$ is obtained by normalizing measured power output from the past $30 \mathrm{~min}$ using clear sky power output. The largest and smallest $k_{\mathrm{cs}}$ are selected for $k_{\mathrm{cs}_{\max }}$ and $k_{\mathrm{cs}_{\min }}$, respectively. If CMVs are not available in the $30 \mathrm{~min}$ search window, typically suggesting (near) clear or overcast conditions, then the WCS-RR is not computed. Fortunately, in uniform sky cover conditions, the PV output variation is small and the associated ramp events are not important for PV planning applications. Finally, since PV data are sampled every $2 \mathrm{~s}$, the WCS-RR is also computed in $2 \mathrm{~s}$ intervals for consistency, but the analysis could be conducted with 15 min or hourly data.

\section{RESULTS AND DISCUSSION}

\section{A. Performance on an example day}

In this section, one detailed example is analyzed to illustrate and explain the performance of the proposed model. The WCS-RR estimate on June 21,2018 , one of the best days (100\% compliance across all evaluation windows, refer to Table II in the Appendix D), is validated against the actual ramp rates in Fig. 4. The top plot shows real PV power from 10:30 to 15:15 PDT and clear sky power output from 2 days earlier. The clouds are observed to move from eastward to southward over the day with speeds ranging from 2 to $5 \mathrm{~m} \mathrm{~s}^{-1}$, as illustrated in the middle plot. The bottom plot illustrates the observed ramp rate and the WCS-RR estimates.

Due to increasing clear sky power and cloud speed, the WCS-RR generally increases with time, completely enveloping the actual ramp

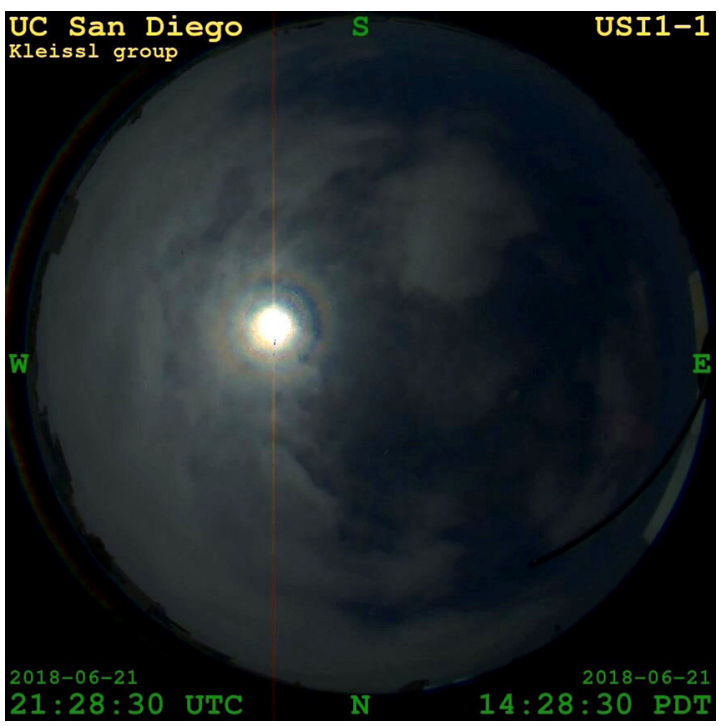

FIG. 5. Sky image of a cloud shading event at 14:28 PDT on June 21, 2018 with the largest ramp of the PV plant. Figure 4 demonstrates that this largest ramp is accurately bracketed by the WCS-RR. 
rate magnitude and timing. Since the cloud optical thickness is steady over the 5-h period, $k_{\mathrm{cs}}$ variation does not have a strong impact on the WCS-RR. There is a 30 min exception around 11:45 PDT when the sky was briefly clear, lowering the WCS-RR estimates and the difference between $k_{\mathrm{cs}_{\max }}$ and $k_{\mathrm{cs}_{\min }}$, but the ramp events in that period are still enveloped successfully. The largest observed ramp rate of the day occurred at 14:28 PDT, during partial cumulus cloud cover (Fig. 5). Because this cloud condition change (clear to worst-case thick clouds) is the exact circumstance modeled in Eq. (6), the WCS-RR estimate accurately captures the ramp magnitude with only a $7 \%$ overestimate.

The daily noncompliance rate of $0 \%$ across all evaluation windows on this day confirms that the observed ramp rates are perfectly enveloped by the WCS-RR, at a cost of averaged ramp rate overestimates over the designated time window from $45 \%$ (30 min window) to $62 \%$ ( 2 min window). The ideal metrics would be $0 \%$ noncompliance and $0 \%$ overestimate. In reality, there is a trade-off between the noncompliance and the overestimate metrics: to bracket all large power ramps, the model conservatively assumes a cloud condition change from clear sky to thick clouds at all times, including periods with clear or overcast skies, which inevitably over-predicts the ramp rates. The only hypothetical scenario with $0 \%$ noncompliance and $0 \%$ overestimate would be for a series of thick clouds of the same size as the PV plant to pass the plant along L (or analogously W); in those conditions, the plant would continually ramp up or down with a ramp rate equal to the WCS-RR. For June 21, 2018, the minimal overprediction of the largest ramp event (on 14:28 PDT) proves that the over-prediction is not excessive. The trade-off between the overestimate and noncompliance rate will be further discussed in Section IV E.

\section{B. Aggregate ramp rate statistics}

The evaluation of the proposed method over an extensive set of 90 days is summarized in Table I, and the performance metrics are illustrated by the box plot in Fig. 6. A more detailed day-by-day performance comparison is tabulated in Appendix D. Overall, the method shows promise: for the shortest 2 min window, the average noncompliance rate is only $1.1 \%$ at a cost of a $64.3 \%$ overestimate. The noncompliance rate slightly worsens with wider evaluation windows, which is expected as the chance that a noncompliance event $(\sigma>1)$ is included in the evaluation window increases with wider windows. Nevertheless, even under the longest $30 \mathrm{~min}$ evaluation window, the average noncompliance rate is only $6 \%$. The largest-ever observed ramp rate of $9.2 \mathrm{~kW}$ (or $29.7 \%$ PV capacity) per second on February 27, 2018 is successfully captured, and on this day, the noncompliance rate of $0 \%$ is achieved across all evaluation windows, further demonstrating that the proposed WCS-RR model functions as designed. It is important to note that the noncompliance rate indicates the occurrence in the percentage of time intervals where the maximum actual

TABLE I. Noncompliance rate $\epsilon$ and the degree of overestimation $\delta$ for $2 \mathrm{~min}$, $10 \mathrm{~min}$, and $30 \mathrm{~min}$ evaluation time windows averaged over all data points in 90 days. For all metrics, small values indicate better performance. Refer to Fig. 6 for the statistical distribution of $\epsilon$ and $\delta$. Refer to Table II for a daily tabulation.

\begin{tabular}{|c|c|c|c|c|c|c|}
\hline \multicolumn{7}{|c|}{$\epsilon_{2 \min }(\%) \delta_{2 \min }(\%) \epsilon_{10 \min }(\%) \delta_{10 \min }(\%) \epsilon_{30 \min }(\%) \delta_{30 \min }(\%)$} \\
\hline Average & 1.1 & 64.3 & 2.9 & 56.8 & 5.9 & 50.4 \\
\hline
\end{tabular}

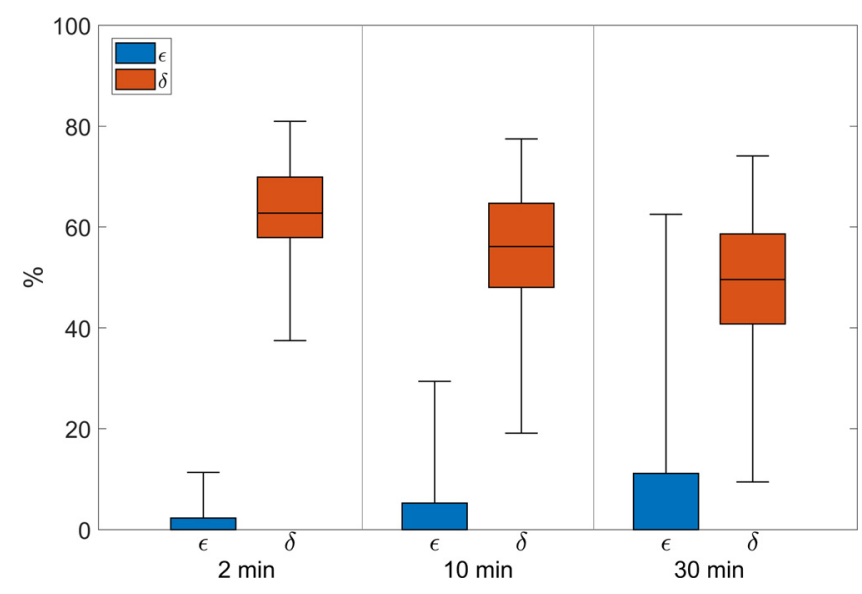

FIG. 6. Box plot of noncompliance rate $\epsilon$ and associated overestimate $\delta$ for 2 min $10 \mathrm{~min}$, and 30 min evaluation time windows over 90 days with the WCS-RR model. This figure is a visual representation of the performance metrics in Table II.

ramp in the evaluation time windows exceeds the corresponding WCS-RR. The degree of overestimation worsens with the shorter evaluation window length (greater number of windows). Because WCS$\mathrm{RR}$ estimates are generally conservative at all times except the time of the daily largest ramp events (e.g., 14:28 PDT in Fig. 4), the degree of overestimate would be minimal when only a single time window (i.e., the daily largest ramp event) is considered over the day but tends to be larger when more evaluation windows are considered.

As the primary goal of the WCS-RR model is to estimate the largest possible ramp rate, the observed maximum ramp rates and associated binary daily success/failure flags are also tabulated for each day in Table II. The WCS-RR successfully envelopes the maximum daily observed ramp events on 83 out of 90 days. For one of the remaining 7 days, the WCS-RR was not computed because CMV measurements were not available at the moment of the largest ramp rate. While ramp rate violations are observed on the other 6 days, the actual ramp rate exceedances are relatively small at $(0.7,0.2,0.1,0.8,0.2,0.3) \mathrm{kW} \mathrm{s}^{-1}$ (refer to the value in the parentheses after the "F" flag in Table II). The causes for these violations are discussed in the Sec. IV C.

For PV system planning applications, statistics about the magnitude and frequency of noncompliance events are of interest. The distributions of the ramp size and exceedance of all 129 noncompliance events $(\sigma>1)$ over the 90 days are displayed in Fig. 7. The left histogram indicates that $92 \%$ of the missed ramps are less than $6 \% \mathrm{~s}^{-1}$. The right histogram indicates that most ramp rate exceedances normalized by the rated $\mathrm{AC}$ power are less than $2 \% \mathrm{~s}^{-1}$. Thus, Fig. 7 illustrates that even when the real ramps exceed the WCS-RR estimate, the difference is small and most of the noncompliance events only involve small ramps compared to the largest observed ramp of $29.7 \% \mathrm{~s}^{-1}$ (February $27,2018)$. While the ramps may seem large for utility-scale plant operators, relative ramp rates decrease with the size of the PV plant. Since Eqs. (5) and (6) have the solar power plant area in the denominatorfor the same irradiance field-for example, ramps for a $371 \mathrm{MW}$ plant would be $1 / 10000$ those of our $37.1 \mathrm{~kW}$ plant. Therefore, for example, the $2 \% \mathrm{~s}^{-1}$ ramps should not be judged as an absolute number, but rather relative to the $29.7 \%$ maximum ramp rate for our particular PV plant. 

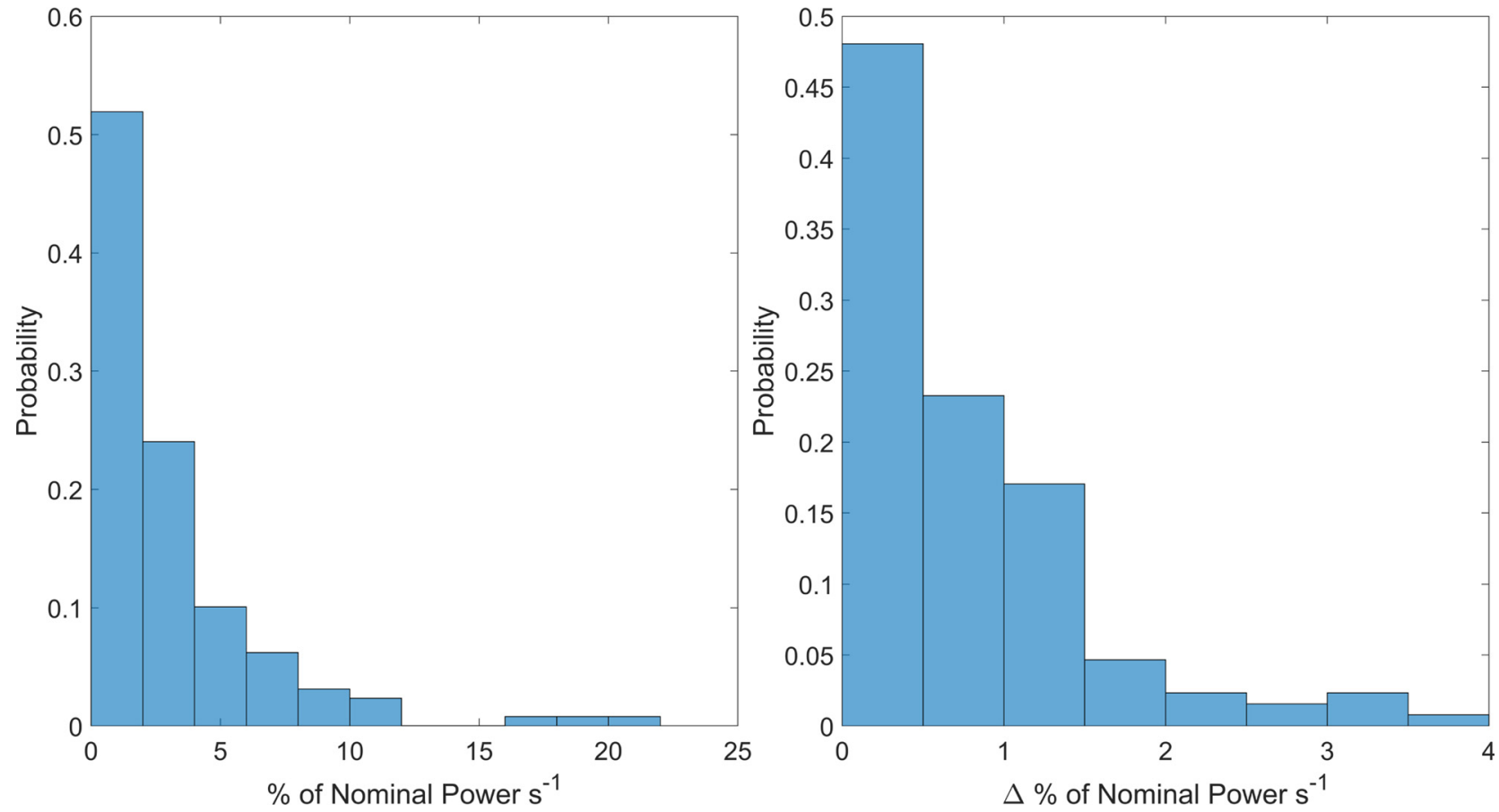

FIG. 7. Distribution of noncompliance events by the ramp size (left) and exceedance (right) over 90 days.

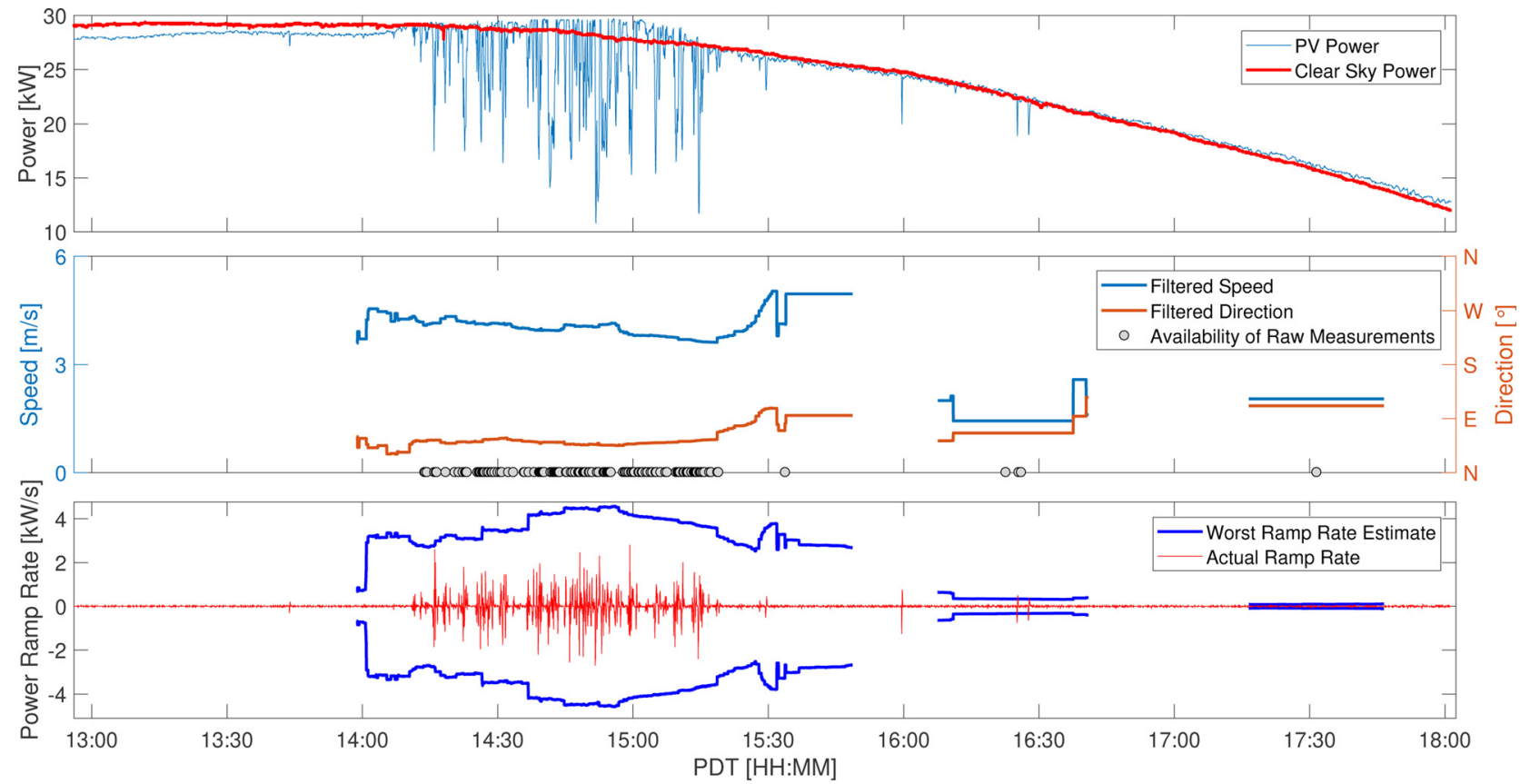

FIG. 8. Example day when the observed ramp does not comply with the computed WCS-RR at 16:25 and 16:28 PDT. Top: real PV power on June 27, 2018 and the most recent clear day. Middle: cloud speed and direction from the CSS. Bottom: comparison between the actual ramp rate and the WCS-RR estimate. 


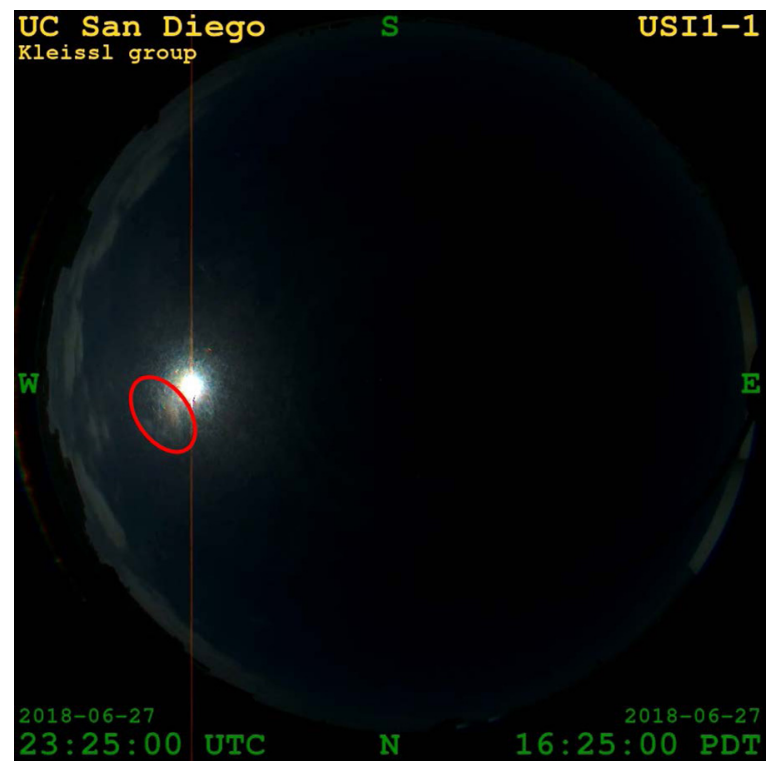

FIG. 9. Sky image of a small and dissipating stratocumulus cloud (red circle) that caused the noncompliance events on 16:25 PDT, June 27, 2018 (Fig. 8).

\section{Example noncompliance events}

In this section, the causes of noncompliance events are further investigated, and the limitations of the model and experimental setup are discussed. June 27, 2018 is analyzed as a representative day with noncompliance events detailed in Fig. 8. Three ramps exceeded the WCS-RR from 16:00 to 16:30 PDT. The WCS-RR did not produce an estimate for the first ramp event and did not bracket the other two. Visual inspection of sky images reveals that the three power ramps were induced by small, dissipating stratocumulus clouds (e.g., red circle in Fig. 9). The cloud cover fraction is likely too small to induce frequent illuminance fluctuations in the CSS, resulting in only two CMV measurements (dots in the middle plot, Fig. 8) in the 30 min window and no measurement at all within $\pm 15 \mathrm{~min}$ of the first ramp event at 16:00 PDT. Furthermore, the cloud is dissipating as it crosses the measurement site, and as a result, the cloud edge is not sharp enough to satisfy the assumption of LCE, which degrades the goodness of the curve fit (Wang et al., 2016). Subsequently, the CMV measurement is likely inaccurate, which, in turn, degrades the accuracy of the WCSRR. Since these shortcomings in the CSS are specific to thin and sporadic clouds, they are not of concern for the main application of the WCS-RR model, which is to estimate the possible maximum ramp events for PV planning. In fact, the maximum ramp event of the day at 14:15 PDT was still successfully bracketed. This example day demonstrates that the accuracy of the proposed method primarily relies on the quality and availability of CMVs.

By examining the sky images for all noncompliance events, we conclude that the primary reasons for noncompliance events are few and/or inaccurate CSS measurements. For example, for many noncompliance events, only one single CMV measurement appeared in the $30 \mathrm{~min}$ search window, which is an insufficient sample size. Sparse CSS measurements are typically caused by sparse cloud fields and are associated with significant uncertainty, which affects the performance

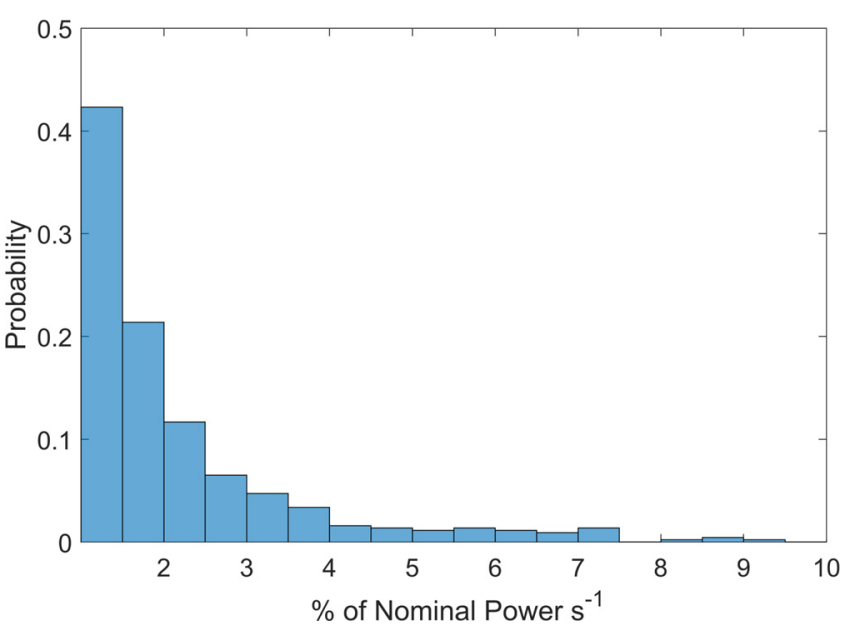

FIG. 10. Distribution of ramp rates over 90 days when none or only one CMV measurement is available in the 30 min search window. Most of these ramp events do not comply with the respective WCS-RR estimates.

of the WCS-RR method because CMV is the key input to the WCSRR [as per Eq. (1)]. Sparse and/or small clouds may even go undetected by the CSS, and thus, a WCS-RR estimate would not be produced. However, these clouds may not be large enough to cause meaningful ramps. Figure 10 shows the distribution of ramp rates for times with one or less CMV measurements in the 30 min search window over the 90 days. All ramp sizes are smaller than $10 \%$ PV capacity per second even for this small PV system, which confirms our expectation. For large PV systems, we expect sparse or small clouds to be even less relevant as they cover only a fraction of the PV plant.

\section{Comparison to other simple ramp rate estimates}

The method provides the reasonable maximum RRs through a simple process model framework, which characterizes the maximum RRs better than other simpler methods (e.g., a constant ratio). For

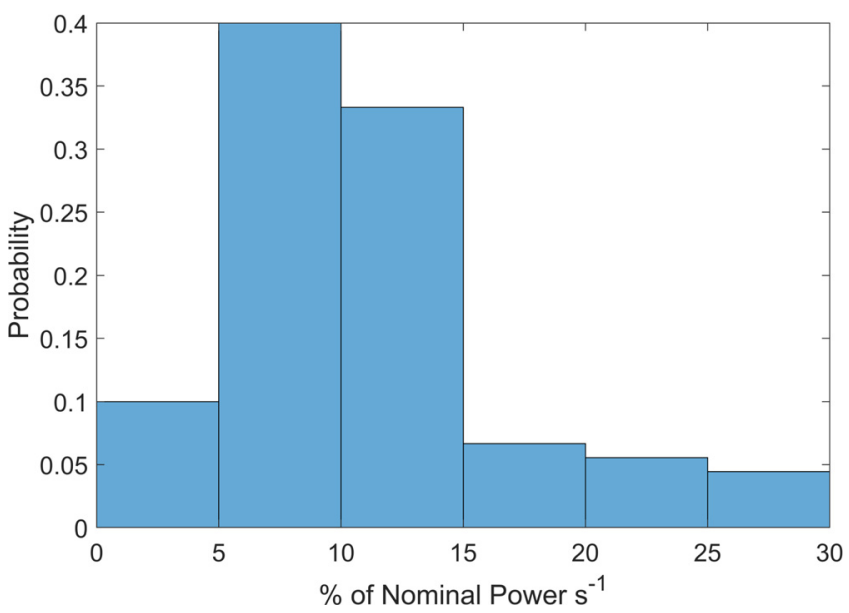

FIG. 11. Distribution of daily maximum $1 \mathrm{~s}$ ramp rates in percentage of nominal power per second over 90 days. 
example, Fig. 11 shows the distribution of the observed daily maximum power ramp rate over 90 days normalized by PV system's nominal power. Based on this histogram, a constant ratio of $30 \% \mathrm{~s}^{-1}$ ramp would be a good assumption for this specific PV system and climate. However, because the maximum ramp rate depends on the system size, orientation, prevailing wind direction and speed, and optical depth of the atmosphere, a universal RR assumption for a PV system is a crude assumption and not likely close to the ground truth unless it is prior known. For example, the PV system dimensions are directly accounted for in our model and they can vary by a factor of 100 more between a commercial rooftop PV system and a utility-scale PV system, resulting in ramps on the order of $10 \% \mathrm{~s}^{-1}$ and $10 \% \mathrm{~min}^{-1}$, respectively.

To point out the value of our proposed method, we also add a simpler assumption of a constant ratio of $70 \% \mathrm{~s}^{-1}$ ramp to the analyses to compare with our method. The box plot of noncompliance rate $\epsilon$ and associated overestimate $\delta$ with the simpler model is presented in Fig. 12. The flat ramp rate of $70 \% \mathrm{~s}^{-1}$ is able to bracket all power ramps of this small PV system at a cost of $>90 \%$ overestimation, while our method yields $<6 \%$ noncompliance rate at a cost of $<60 \%$ overestimation (Fig. 6). Thus, a conservative constant maximum ramp assumption (to avoid any noncompliance) would result in a significantly larger degree of overestimation than the WCS-RR. Conversely, a small constant maximum ramp assumption may result in a large noncompliance rate. The advantage of our method is that as long as basic historical data for a given site are available, the maximum ramp rate can be estimated. The trade-off between noncompliance and the degree of overestimation is expected and further discussed in Section IV E.

On the other hand, if a simpler threshold was chosen, the energy storage system would be linearly over/undersized according to the ratio of the chosen maximum ramp rate to the optimal maximum ramp rate. Using our method, the energy storage system can be sized appropriately.

\section{E. Tradeoff between noncompliance and the degree of overestimation}

The trade-off between the noncompliance rate and the degree of overestimation deserves further discussion. For example, an energy

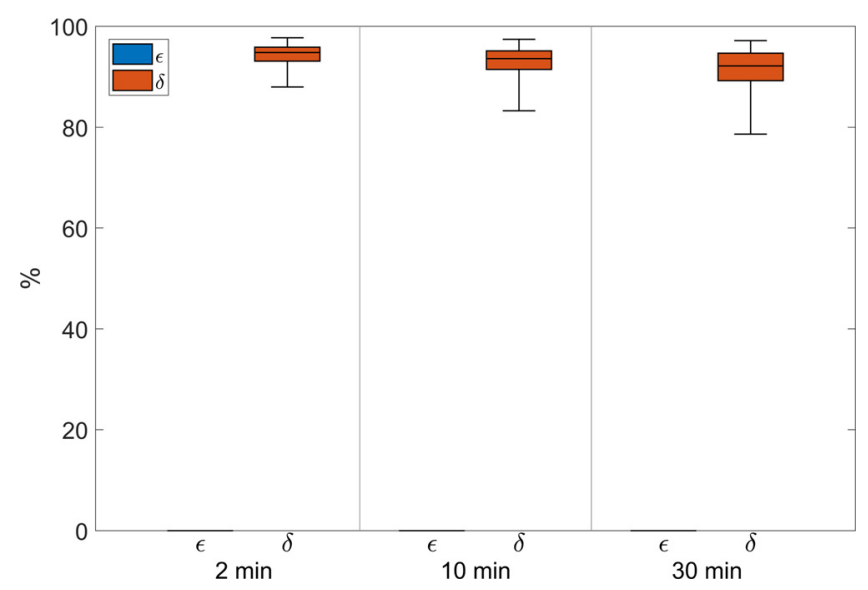

FIG. 12. Box plot of noncompliance rate $\epsilon$ and associated overestimate $\delta$ for 2 min, $10 \mathrm{~min}$, and 30 min evaluation time windows over 90 days with a simpler model of constant ramp rate of $70 \% \mathrm{~s}^{-1}$. storage system that can mitigate larger ramp rates is more costly, but a smaller energy storage system may not mitigate all ramps and result in noncompliance penalties and/or curtailed solar energy. Ultimately, economic modeling specific to a project is needed to answer these questions. In such conditions, a safety factor could be introduced in Eq. (5) to determine appropriate $k_{\mathrm{Cs}_{\mathrm{i}}}$ and $k_{\mathrm{Cs}_{\mathrm{o}}}$ and accordingly adjust the WCS-RR to fit the risk profile of the investor. Finally, the possible ways to reduce the amount of prediction overhead include the following: (1) PV performance models will be needed for more accurate $k_{\mathrm{cs}}$ measurements. (2) The power mismatch needs to be considered. For example, the wiring diagram of the PV system can be integrated in the model to count for the extra power loss depending on in which direction the cloud shadow is intercepting the solar arrays.

\section{F. Applications with low-frequency solar irradiance data}

The proposed method is universally applicable even in the absence of local cloud speed or $k_{\mathrm{cs}}$ measurements since the cloud velocity can be derived from NWP data, such as the North American Mesoscale Forecast System (NAM), which contains surface solar irradiance, cloud cover (or at least relative humidity), and wind vectors at different pressure levels, among other variables (see, e.g., Lave and Kleissl, 2013; Pelland et al., 2013). The Solar Integration National Dataset (SIND) (https://www.nrel.gov/grid/sind-toolkit.html, accessible as of August, 2020) from the NREL is also an alternative source for solar irradiance data (Lave and Weekley, 2016). Finally, as discussed earlier, for higher model accuracy, $k_{\mathrm{cs}}$ can be obtained from PV performance models vs the simpler heuristic method used in this paper.

\section{G. Benefits for PV plant sizing and energy storage sizing and scheduling}

The proposed WCS-RR model reveals that, in addition to the meteorology (cloud motion and optical depth), the PV plant shape and orientation with respect to the prevailing winds at the cloud level can significantly impact the maximum power ramp rate. Our results may inspire PV plant designers to preferentially select plant orientations that result in smaller ramp rates depending on the dimension of the PV system relative to the typical cloud scale at a given location. For example, if consistent prevailing winds at the cloud level are observed as westward or eastward, then extending the PV plant in the east-west direction reduces the relative ramp rates. With an optimal PV plant orientation, the PV system operator can smooth the power output variability by using a smaller ESS. Note that prevailing winds may change seasonally, resulting in potential trade-offs between peak and off-peak production. Furthermore, if cloud sizes are very small relative to the PV plant size, then the PV plant orientation relative to the wind direction becomes less important, as shown in Arias-Castro et al. (2014).

During operation, the WCS-RR would be useful to hour ahead or day ahead strategic bidding in energy markets because generator revenues are related to both the price-energy curve and the expected ramp rates. If solar forecasts suggested thin clouds, slow clouds, or a favorable cloud movement direction, less of the ESS power and energy would have to be reserved for ramp rate control and more could be utilized to monetize other value streams. The WCS-RR would also be useful to ESS operation for benefit stacking (e.g., frequency regulation and ramp rate mitigation), as less energy and power capacity of the 
ESS would have to be reserved for ramp rate compliance. Similarly, during ESS planning, if the WCS-RR is smaller at a site given historical weather data, the ESS size could be reduced without risking a ramp rate violation.

\section{CONCLUSIONS}

Knowing the maximum expected photovoltaic (PV) production ramp rate proves to be useful for the design of $\mathrm{PV}$ and energy storage systems (ESSs) by determining the ESS energy reserve required to offset power fluctuations. The main goal of this paper is to provide a method to inform optimal design of a solar power plant with ESSs by estimating the worst-case scenario ramp rate (WCS-RR) in the design phase, prior to the PV installation and in the absence of local high-frequency irradiance data. A method to estimate the WCS-RR for a PV plant, based on the cloud speed and direction, solar irradiance (or power), and geometric PV plant layout is developed and validated. The principal assumption is that the cloud field properties are stationary during the cloud passage over the plant. The WCS-RR is validated against a PV system during a 10 -month period, using cloud motion vectors (CMVs) measured by a cloud speed sensor (CSS). The largest observed ramp of $29.7 \% \mathrm{~s}^{-1}$ is contained with the worst case estimate of $34.3 \% \mathrm{~s}^{-1}$. The actual ramp rates comply with the calculated maximum ramp rates $98.9 \%$ of the time with a 2 min evaluation time window. The remaining $1.1 \%$ of times can be primarily ascribed to inaccurate cloud velocity measurements in conditions with sparse and/or thin clouds. The high compliance rate also indirectly demonstrates the accuracy of our recently remodeled CSS. Our WCS-RR method will be helpful for both PV plant planning and operation.

\section{ACKNOWLEDGMENTS}

Juan Bosch was financed in part by Project No. PID2019108953RB-C21, funded by the Ministerio de Ciencia e Innovación and co-financed by the European Regional Development Fund. In addition, Inigo de la Parra was partially supported by the Spanish State Research Agency (AEI) and FEDER-UE under Grant Nos. DPI2016-80641-R and DPI2016-80642-R. Finally, we are grateful for Minghua Ong's editorial support and Dr. Shuang Cui's drone photography assistance.

\section{APPENDIX A: OUTPUT SAMPLING RATE REQUIREMENT}

A physical limit for the validation of the WCS-RR method is that its sampling time needs to be such that the cloud velocity times the sampling time step does not exceed the dimension of the PV system in the direction of cloud motion (note: the application of the WCS-RR does not require high sampling frequency data). Geometrically, Eq. (1) in this paper is only valid if the following constraint is met:

$$
\Delta t \leq \min \left\{\frac{\mathrm{W}}{|\cos \alpha| v}, \frac{\mathrm{L}}{|\sin \alpha| v}\right\} .
$$

For any given sampling rate and dimension of the PV system, there is a cloud velocity for which that sampling rate starts to become too slow, and the WCS-RR then incorrectly predicts the

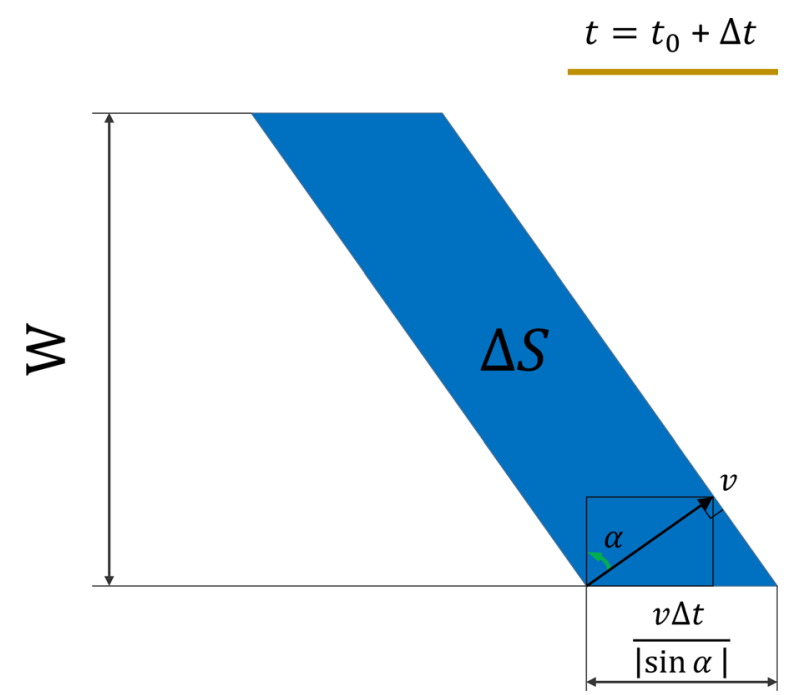

FIG. 13. Trigonometric diagram of the effectively impacted area $\Delta S$ discussed in Fig. 1 (b). Note for illustration purposes, $\alpha$ in this diagram does not align with Fig. 1.

ramp rate over the sampling time step. Since the cloud velocity has a physical limit, a conservative sampling rate can be derived given the dimension of the PV system. Large PV systems can tolerate larger sampling rates. For example, assume that a typical PV panel with a power rating of 208 watts measures $1.6 \mathrm{~m} \times 1 \mathrm{~m}$ for $1.6 \mathrm{~m}^{2}$ of area. A $5 \mathrm{MW} \mathrm{PV} \mathrm{system} \mathrm{with} 24000 \mathrm{PV}$ panels and a typical ground coverage ratio of 0.6 would then cover a square measuring $320 \mathrm{~m}$ on each side (including $200 \mathrm{~m}$ spacing). With $\alpha$ ranging from 0 to $90^{\circ}, v=25 \mathrm{~m} / \mathrm{s}$, and $\mathrm{W}=\mathrm{L}=320 \mathrm{~m}$, a sampling time step of $13 \mathrm{~s}-18 \mathrm{~s}$ (or shorter) will be needed depending on the specific cloud direction. In summary, a sampling time step of $13 \mathrm{~s}$ will guarantee that our model is geometrically valid for a $5 \mathrm{MW}$ PV system.

\section{APPENDIX B: TRIGONOMETRIC DIAGRAM OF EFFECTIVELY AFFECTED AREA $\Delta S$}

The derivation of the effectively impacted area can be formulated as $\Delta S=\mathrm{W} v \Delta t /|\sin \alpha|$ per Fig. 13 .

Note that $\Delta S$ is always less than or equal to $\Delta S_{\mathrm{i}}$. Moreover, this formula is derived assuming that the cloud boundary initially covers the $\mathrm{W}$ side. If the cloud boundary initially covers the $\mathrm{L}$ side (per Fig. 1), then $\Delta S=\mathrm{L} v \Delta t /|\cos \alpha|$. Nevertheless, we use the full impacted areas $\Delta S_{\mathrm{i}}$ and $\Delta S_{\mathrm{o}}$ for the calculation of the power changes under all cloud passage circumstances, as indicated in Eq. (6).

\section{APPENDIX C: PHASES OF CLOUD PASSAGE VS EFFECTIVELY AFFECTED AREA $\Delta S$}

The WCS-RR model only considers the maximum expected ramp rate in two ways: (i) a clear sky is giving way to an overcast sky; (ii) considering the time when the effectively affected area reaches the maximum during the passage of cloud. For 


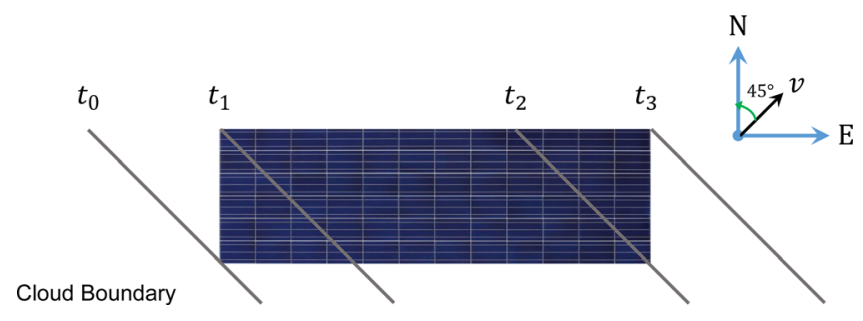

FIG. 14. Schematic of the three phases of power changes during the course of cloud passage with cloud speed of $0.5 \mathrm{~m} / \mathrm{s}$ and cloud direction of $45^{\circ}$. The PV system measures $200 \mathrm{~m} \times 60 \mathrm{~m}$.

clarification, the phases of cloud passage vs $\Delta S$ are characterized in this section.

For example, assume a PV system measures $200 \mathrm{~m} \times 60 \mathrm{~m}$. A cloud boundary with $\alpha=45^{\circ}$ and $v=0.5 \mathrm{~m} / \mathrm{s}$ starts to shade the PV system at $t_{0}$ from the south-west left corner. In phase I (between $t_{0}$ and $\left.t_{1}\right), \Delta S$ starts from 0 and increases with time because subsequently more incremental areas are covered by the moving cloud, resulting in an increasing power ramp rate. In phase II (between $t_{1}$ and $t_{2}$ ), since the cloud boundary passes the north-west corner at $t_{1}, \Delta S$ reaches its maximum and becomes constant with time, and as a result, the ramp rate reaches a steady state. The worst case scenario occurs during this phase. In phase III (between $t_{2}$ and $t_{3}$ ), the cloud boundary passes the south-east corner of the system. Then, the ramp rate decreases as less subsequently incremental area is covered by the moving cloud until it fully covers the plant at $t_{3}$,
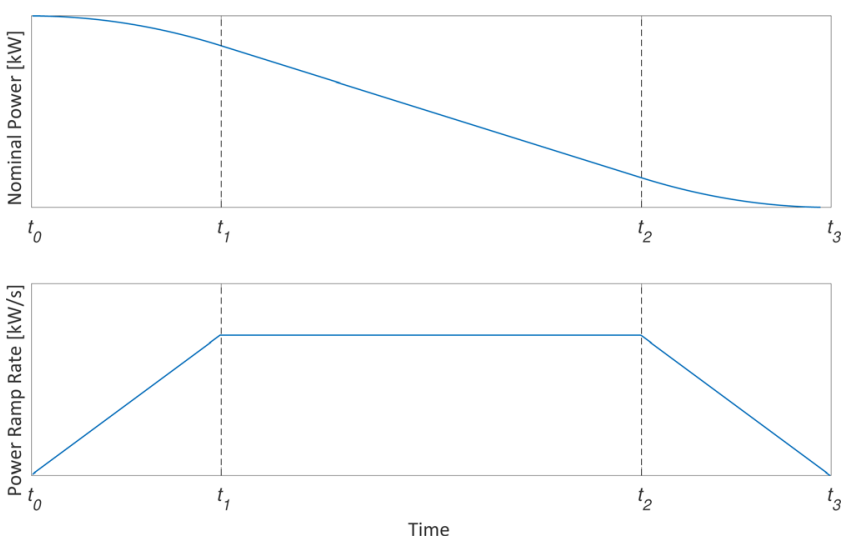

FIG. 15. The nominal power (upper) and ramp rate (lower) in the three phases corresponding to the scenarios and phases of a cloud shading event conceptualized in Fig. 14. The nominal power is calculated as a linear function of the effectively affected area $\Delta S$, while the ramp rate is computed by taking the first order difference of the nominal power. The observed variations in the two subplots illustrate how different phases of cloud passage translate to ramp rates.

after which $\Delta S$ as well as the power variability becomes zero. The three-phase cloud passage and power ramp rates are conceptualized in Fig. 14 and simulated in Fig. 15, respectively. In this paper, we consider phase II in our WCS-RR model to warrant the worst case ramp rate.

\section{APPENDIX D: DAY-BY-DAY PERFORMANCE COMPARISON TABLE}

TABLE II. Daily ramping duration (when more than $1 \% \mathrm{~s}^{-1}$ power variation is present), absolute value of daily observed maximum ramp rate in $\mathrm{kW} \mathrm{s}^{-1}$, success of bracketing the maximum ramp rate, noncompliance rate $\epsilon$, and associated degree of overestimation $\delta$ for $2 \mathrm{~min}, 10 \mathrm{~min}$, and $30 \mathrm{~min}$ evaluation time windows for 90 days. The average in the last row is computed over all data points. Small values of $\epsilon$ and $\delta$ indicate better performance.

\begin{tabular}{|c|c|c|c|c|c|c|c|c|c|}
\hline Date & $\begin{array}{l}\text { Daily } \\
\text { ramping } \\
\text { duration } \\
\text { (min) }\end{array}$ & $\begin{array}{l}\text { Absolute } \\
\text { maximum } \\
\text { ramp rate } \\
\left(\mathrm{kW} \mathrm{s}^{-1}\right)\end{array}$ & $\begin{array}{c}\text { Success }(\mathrm{S}) \text { or failure }(\mathrm{F}) \\
\text { of bracketing daily } \\
\text { maximum ramp rate, } \\
\text { (ramp rate exceedance in } \Delta \mathrm{kW} \mathrm{s}^{-1} \text { ) }\end{array}$ & $\epsilon_{2 \min }(\%)$ & $\delta_{2 \min }(\%)$ & $\epsilon_{10 \min }(\%)$ & $\delta_{10 \min }(\%)$ & $\epsilon_{30 \min }(\%)$ & $\delta_{30 \min }(\%)$ \\
\hline $10 / 8 / 17$ & 275 & 3.7 & S & 0.0 & 64.4 & 0.0 & 57.4 & 0.0 & 52.2 \\
\hline $10 / 10 / 17$ & 140 & 4.2 & S & 11.3 & 37.5 & 29.4 & 19.1 & 62.5 & 9.4 \\
\hline $10 / 11 / 17$ & 455 & 4.4 & S & 0.5 & 58.8 & 2.1 & 46.2 & 5.9 & 35.8 \\
\hline $10 / 13 / 17$ & 115 & 1.4 & S & 4.5 & 47.7 & 13.3 & 39.0 & 28.6 & 40.3 \\
\hline $11 / 26 / 17$ & 125 & 2.4 & S & 3.7 & 56.9 & 13.3 & 48.6 & 28.6 & 44.3 \\
\hline $12 / 3 / 17$ & 230 & 2.1 & S & 0.0 & 61.3 & 0.0 & 52.4 & 0.0 & 42.4 \\
\hline $12 / 16 / 17$ & 180 & 3.1 & S & 0.0 & 66.2 & 0.0 & 61.4 & 0.0 & 54.3 \\
\hline $1 / 9 / 18$ & 170 & 9.0 & S & 1.4 & 68.8 & 5.0 & 60.8 & 14.3 & 49.6 \\
\hline $1 / 10 / 18$ & 55 & 1.8 & $\mathrm{~F}(0.7)$ & 11.1 & 68.9 & 22.2 & 67.6 & 20.0 & 62.7 \\
\hline $1 / 12 / 18$ & 35 & 2.2 & $S$ & 0.0 & 49.8 & 0.0 & 43.0 & 0.0 & 37.1 \\
\hline $1 / 16 / 18$ & 230 & 2.3 & S & 1.2 & 74.7 & 3.7 & 67.9 & 9.1 & 56.9 \\
\hline $1 / 18 / 18$ & 20 & 2.8 & S & 11.1 & 55.1 & 25.0 & 46.3 & 33.3 & 50.7 \\
\hline $1 / 20 / 18$ & 155 & 4.3 & S & 0.0 & 69.9 & 0.0 & 64.8 & 0.0 & 59.9 \\
\hline $2 / 5 / 18$ & 90 & 2.8 & S & 0.0 & 65.3 & 0.0 & 56.8 & 0.0 & 47.7 \\
\hline $2 / 10 / 18$ & 145 & 3.3 & S & 0.0 & 68.4 & 0.0 & 64.7 & 0.0 & 53.8 \\
\hline
\end{tabular}


TABLE II. (Continued.)

\begin{tabular}{|c|c|c|c|c|c|c|c|c|c|}
\hline Date & $\begin{array}{l}\text { Daily } \\
\text { ramping } \\
\text { duration } \\
(\min )\end{array}$ & $\begin{array}{l}\text { Absolute } \\
\text { maximum } \\
\text { ramp rate } \\
\left(\mathrm{kW} \mathrm{s}^{-1}\right)\end{array}$ & $\begin{array}{c}\text { Success }(\mathrm{S}) \text { or failure }(\mathrm{F}) \\
\text { of bracketing daily } \\
\text { maximum ramp rate, } \\
\text { (ramp rate exceedance in } \Delta \mathrm{kW} \mathrm{s}^{-1} \text { ) }\end{array}$ & $\epsilon_{2 \min }(\%)$ & $\delta_{2 \min }(\%)$ & $\epsilon_{10 \min }(\%)$ & $\delta_{10 \min }(\%)$ & $\epsilon_{30 \min }(\%)$ & $\delta_{30 \min }(\%)$ \\
\hline $2 / 11 / 18$ & 85 & 3.1 & S & 0.0 & 68.5 & 0.0 & 61.3 & 0.0 & 56.7 \\
\hline $2 / 13 / 18$ & 210 & 5.6 & $S$ & 5.7 & 65.9 & 12.5 & 58.8 & 27.3 & 54.7 \\
\hline $2 / 14 / 18$ & 100 & 2.9 & $S$ & 0.0 & 76.7 & 0.0 & 69.2 & 0.0 & 64.2 \\
\hline $2 / 22 / 18$ & 60 & 8.8 & $S$ & 0.0 & 67.1 & 0.0 & 60.9 & 0.0 & 50.6 \\
\hline $2 / 23 / 18$ & 200 & 6.9 & $S$ & 0.0 & 67.1 & 0.0 & 61.1 & 0.0 & 51.2 \\
\hline $2 / 27 / 18$ & 185 & 9.2 & $S$ & 0.0 & 71.7 & 0.0 & 64.8 & 0.0 & 60.7 \\
\hline $3 / 3 / 18$ & 200 & 3.2 & S & 0.0 & 81.0 & 0.0 & 76.1 & 0.0 & 71.7 \\
\hline $3 / 4 / 18$ & 130 & 2.6 & S & 2.6 & 61.0 & 5.0 & 56.6 & 9.1 & 52.7 \\
\hline $3 / 8 / 18$ & 275 & 3.2 & S & 0.0 & 75.8 & 0.0 & 72.6 & 0.0 & 65.1 \\
\hline $3 / 9 / 18$ & 200 & 2.6 & S & 0.0 & 75.0 & 0.0 & 70.7 & 0.0 & 63.0 \\
\hline $3 / 11 / 18$ & 295 & 6.4 & S & 0.0 & 69.6 & 0.0 & 61.2 & 0.0 & 57.3 \\
\hline $3 / 12 / 18$ & 190 & 1.4 & S & 0.0 & 77.4 & 0.0 & 75.2 & 0.0 & 72.1 \\
\hline $3 / 13 / 18$ & 60 & 1.1 & S & 0.0 & 74.1 & 0.0 & 71.9 & 0.0 & 72.3 \\
\hline $3 / 14 / 18$ & 315 & 3.4 & S & 0.0 & 76.3 & 0.0 & 70.0 & 0.0 & 65.4 \\
\hline $3 / 17 / 18$ & 295 & 6.3 & S & 0.0 & 73.3 & 0.0 & 65.4 & 0.0 & 56.7 \\
\hline $3 / 18 / 18$ & 295 & 2.2 & S & 0.0 & 76.2 & 0.0 & 71.2 & 0.0 & 65.2 \\
\hline $3 / 20 / 18$ & 340 & 5.3 & S & 0.0 & 78.4 & 0.0 & 72.7 & 0.0 & 65.6 \\
\hline $3 / 23 / 18$ & 210 & 3.2 & S & 0.0 & 70.0 & 0.0 & 65.7 & 0.0 & 59.1 \\
\hline $3 / 24 / 18$ & 265 & 6.6 & S & 0.0 & 67.1 & 0.0 & 59.7 & 0.0 & 51.0 \\
\hline $3 / 25 / 18$ & 120 & 3.2 & S & 2.3 & 65.8 & 6.7 & 63.6 & 14.3 & 49.8 \\
\hline $3 / 30 / 18$ & 180 & 2.9 & $\mathrm{~F}(0.2)$ & 5.2 & 58.9 & 15.8 & 50.5 & 37.5 & 37.5 \\
\hline $3 / 31 / 18$ & 195 & 0.9 & S & 0.0 & 74.6 & 0.0 & 72.7 & 0.0 & 68.9 \\
\hline $4 / 1 / 18$ & 170 & 2.2 & S & 0.0 & 66.4 & 0.0 & 59.1 & 0.0 & 51.3 \\
\hline $4 / 3 / 18$ & 250 & 3.4 & $S$ & 0.0 & 57.7 & 0.0 & 48.3 & 0.0 & 45.2 \\
\hline $4 / 12 / 18$ & 60 & 3.3 & $\mathrm{~F}(0.1)$ & 5.6 & 74.4 & 11.1 & 67.2 & 16.7 & 59.0 \\
\hline $4 / 19 / 18$ & 345 & 8.5 & $\mathrm{~S}$ & 5.2 & 64.2 & 7.0 & 56.2 & 11.1 & 49.6 \\
\hline $4 / 24 / 18$ & 125 & 2.8 & $\mathrm{~S}$ & 2.6 & 55.3 & 5.3 & 51.5 & 7.1 & 49.3 \\
\hline $5 / 8 / 18$ & 105 & 1.7 & N/A & 0.0 & 67.8 & 0.0 & 61.9 & 0.0 & 58.6 \\
\hline $5 / 9 / 18$ & 150 & 2.5 & $\mathrm{~S}$ & 5.3 & 53.0 & 15.8 & 41.8 & 28.6 & 28.9 \\
\hline $5 / 10 / 18$ & 100 & 2.4 & $\mathrm{~S}$ & 0.0 & 70.7 & 0.0 & 63.7 & 0.0 & 58.8 \\
\hline $5 / 15 / 18$ & 130 & 2.7 & $\mathrm{~S}$ & 2.6 & 51.0 & 5.3 & 42.1 & 8.3 & 40.8 \\
\hline $5 / 17 / 18$ & 395 & 5.3 & $\mathrm{~S}$ & 0.0 & 60.2 & 0.0 & 47.9 & 0.0 & 40.5 \\
\hline $5 / 22 / 18$ & 225 & 2.8 & $\mathrm{~S}$ & 0.0 & 71.5 & 0.0 & 66.7 & 0.0 & 59.7 \\
\hline $5 / 23 / 18$ & 200 & 4.0 & $S$ & 0.0 & 56.8 & 0.0 & 42.4 & 0.0 & 33.1 \\
\hline $5 / 25 / 18$ & 320 & 3.6 & $\mathrm{~S}$ & 0.0 & 63.6 & 0.0 & 53.1 & 0.0 & 46.7 \\
\hline $5 / 26 / 18$ & 335 & 5.8 & $S$ & 0.8 & 65.4 & 2.2 & 57.2 & 5.0 & 49.5 \\
\hline $5 / 27 / 18$ & 160 & 2.2 & $S$ & 0.0 & 66.0 & 0.0 & 58.8 & 0.0 & 54.6 \\
\hline $5 / 28 / 18$ & 165 & 4.6 & $S$ & 0.0 & 59.8 & 0.0 & 48.5 & 0.0 & 34.2 \\
\hline $5 / 30 / 18$ & 215 & 4.4 & $S$ & 1.3 & 62.8 & 3.7 & 55.5 & 7.7 & 44.4 \\
\hline $5 / 31 / 18$ & 225 & 3.9 & $S$ & 3.5 & 56.8 & 10.7 & 48.0 & 25.0 & 47.9 \\
\hline $6 / 2 / 18$ & 95 & 1.4 & $S$ & 0.0 & 52.4 & 0.0 & 43.6 & 0.0 & 35.0 \\
\hline $6 / 3 / 18$ & 260 & 3.3 & $S$ & 2.9 & 58.2 & 6.5 & 48.8 & 14.3 & 41.9 \\
\hline $6 / 5 / 18$ & 245 & 4.0 & $S$ & 0.0 & 58.9 & 0.0 & 48.1 & 0.0 & 42.7 \\
\hline $6 / 10 / 18$ & 95 & 3.0 & $S$ & 0.0 & 57.9 & 0.0 & 44.5 & 0.0 & 30.4 \\
\hline $6 / 21 / 18$ & 245 & 3.0 & $S$ & 0.0 & 62.0 & 0.0 & 53.0 & 0.0 & 45.2 \\
\hline $6 / 27 / 18$ & 85 & 2.8 & $S$ & 5.4 & 60.0 & 10.0 & 48.9 & 20.0 & 44.1 \\
\hline
\end{tabular}


TABLE II. (Continued.)

\begin{tabular}{|c|c|c|c|c|c|c|c|c|c|}
\hline Date & $\begin{array}{l}\text { Daily } \\
\text { ramping } \\
\text { duration } \\
(\min )\end{array}$ & $\begin{array}{l}\text { Absolute } \\
\text { maximum } \\
\text { ramp rate } \\
\left(\mathrm{kW} \mathrm{s}^{-1}\right)\end{array}$ & $\begin{array}{c}\text { Success }(\mathrm{S}) \text { or failure }(\mathrm{F}) \\
\text { of bracketing daily } \\
\text { maximum ramp rate, } \\
\left(\text { ramp rate exceedance in } \Delta \mathrm{kW} \mathrm{s}^{-1} \text { ) }\right.\end{array}$ & $\epsilon_{2 \min }(\%)$ & $\delta_{2 \min }(\%)$ & $\epsilon_{10 \min }(\%)$ & $\delta_{10 \min }(\%)$ & $\epsilon_{30 \min }(\%)$ & $\delta_{30 \min }(\%)$ \\
\hline $6 / 28 / 18$ & 140 & 3.1 & S & 0.0 & 60.6 & 0.0 & 48.8 & 0.0 & 34.7 \\
\hline $6 / 29 / 18$ & 380 & 4.6 & $S$ & 0.0 & 58.6 & 0.0 & 47.0 & 0.0 & 41.0 \\
\hline $6 / 30 / 18$ & 320 & 3.8 & $S$ & 1.6 & 59.7 & 5.1 & 51.3 & 5.9 & 48.2 \\
\hline $7 / 4 / 18$ & 70 & 2.9 & $S$ & 0.0 & 55.0 & 0.0 & 37.3 & 0.0 & 24.9 \\
\hline $7 / 13 / 18$ & 150 & 2.1 & $S$ & 1.7 & 59.2 & 5.3 & 53.6 & 11.1 & 49.5 \\
\hline $7 / 14 / 18$ & 200 & 3.5 & $S$ & 6.2 & 60.5 & 12.0 & 48.3 & 18.2 & 45.0 \\
\hline $7 / 15 / 18$ & 120 & 2.5 & S & 0.0 & 52.8 & 0.0 & 39.5 & 0.0 & 27.6 \\
\hline $7 / 17 / 18$ & 95 & 1.1 & S & 0.0 & 79.5 & 0.0 & 77.5 & 0.0 & 74.1 \\
\hline $7 / 18 / 18$ & 265 & 2.9 & S & 0.0 & 74.0 & 0.0 & 68.1 & 0.0 & 60.8 \\
\hline $7 / 20 / 18$ & 365 & 3.6 & S & 0.0 & 61.0 & 0.0 & 52.9 & 0.0 & 46.8 \\
\hline $7 / 21 / 18$ & 465 & 6.1 & $\mathrm{~F}(0.8)$ & 0.5 & 61.1 & 1.9 & 54.2 & 5.0 & 46.4 \\
\hline $7 / 22 / 18$ & 115 & 3.5 & $S$ & 0.0 & 60.8 & 0.0 & 52.9 & 0.0 & 41.1 \\
\hline $7 / 26 / 18$ & 165 & 2.4 & S & 1.7 & 55.5 & 5.0 & 42.0 & 10.0 & 33.1 \\
\hline $7 / 27 / 18$ & 75 & 2.0 & S & 0.0 & 67.4 & 0.0 & 63.5 & 0.0 & 61.8 \\
\hline $7 / 28 / 18$ & 130 & 2.7 & S & 0.0 & 56.9 & 0.0 & 44.5 & 0.0 & 38.7 \\
\hline $7 / 29 / 18$ & 65 & 1.2 & S & 5.6 & 55.7 & 9.1 & 53.6 & 12.5 & 52.0 \\
\hline $7 / 31 / 18$ & 40 & 1.6 & $\mathrm{~F}(0.2)$ & 10.0 & 46.2 & 14.3 & 34.9 & 16.7 & 40.2 \\
\hline $8 / 12 / 18$ & 80 & 1.7 & S & 5.3 & 62.7 & 10.0 & 54.1 & 16.7 & 48.6 \\
\hline $8 / 13 / 18$ & 190 & 6.6 & $\mathrm{~F}(0.3)$ & 2.5 & 53.5 & 10.0 & 41.1 & 28.6 & 31.6 \\
\hline $8 / 14 / 18$ & 390 & 5.8 & $S$ & 1.9 & 60.6 & 7.1 & 47.9 & 13.3 & 36.2 \\
\hline $8 / 20 / 18$ & 145 & 3.1 & $S$ & 0.0 & 74.9 & 0.0 & 69.6 & 0.0 & 64.5 \\
\hline $8 / 21 / 18$ & 35 & 1.0 & S & 0.0 & 62.1 & 0.0 & 59.4 & 0.0 & 59.1 \\
\hline $8 / 23 / 18$ & 290 & 4.1 & S & 0.0 & 58.7 & 0.0 & 45.6 & 0.0 & 36.5 \\
\hline $8 / 24 / 18$ & 185 & 2.2 & S & 0.0 & 70.9 & 0.0 & 63.1 & 0.0 & 53.3 \\
\hline $8 / 25 / 18$ & 140 & 2.6 & S & 7.3 & 50.8 & 16.7 & 39.6 & 27.3 & 40.8 \\
\hline $8 / 26 / 18$ & 105 & 2.6 & S & 0.0 & 52.7 & 0.0 & 46.5 & 0.0 & 40.0 \\
\hline $8 / 27 / 18$ & 245 & 1.5 & S & 0.0 & 75.0 & 0.0 & 65.1 & 0.0 & 54.8 \\
\hline $8 / 28 / 18$ & 145 & 2.2 & S & 0.0 & 56.6 & 0.0 & 56.0 & 0.0 & 48.5 \\
\hline $8 / 29 / 18$ & 185 & 3.1 & S & 0.0 & 69.0 & 0.0 & 63.0 & 0.0 & 58.0 \\
\hline Average & N/A & N/A & $83 \mathrm{~S} / 90$ & 1.1 & 64.3 & 2.9 & 56.8 & 5.9 & 50.4 \\
\hline
\end{tabular}

\section{APPENDIX E: PROCEDURE OF DETERMINING NONCOMPLIANCE RATE $\epsilon$}

Figure 16 illustrates the evaluation process of noncompliance rate $\epsilon$ with a time window of $30 \mathrm{~min}$ on October 10, 2017 (Table II). On this day, the overall time span of the power data is divided into 8 windows (indicated by the dashed lines) with 30 min time intervals each. In each window, the maximum actual ramp rate is compared to the corresponding WCS-RR estimate. If the maximum actual ramp rate complies with the WCS-RR estimate, then the compliance requirement is met and the window is marked in green; otherwise, the window is marked in red. As introduced in Section II C, the noncompliance rate is defined by the number of red labeled windows normalized by the total number of windows. In this case, 5 out of 8 windows are marked in red, yielding a $62.5 \%$ noncompliance rate. However, Fig. 16 shows that overall only 7 actual ramp rates in all intervals do not comply with the WCS-RR estimates. Furthermore, the daily maximum ramp rate at 14:15 PST was successfully enveloped by the corresponding WCS-RR estimate. We proposed the non-compliance metric as defined in Eq. (10) because it is most important in practice that the local or daily maximum ramp rates comply with the corresponding WCS-RR estimates. If we considered all power ramps in the evaluation, we would always achieve a very low noncompliance rate, preventing us from assessing the true performance of our proposed method. 


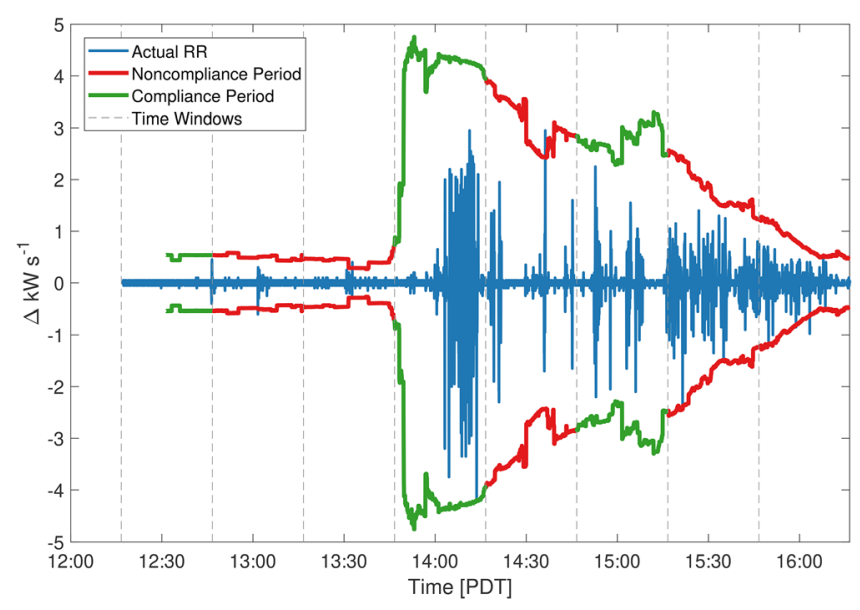

FIG. 16. Example noncompliance evaluation of the proposed method for $30 \mathrm{~min}$ time intervals on Oct. 10, 2017. The actual ramp rates are presented in blue. The time period with power fluctuations is divided into eight local windows with a window length of $30 \mathrm{~min}$. The WCS-RR is coated in green if the maximum actual ramp rate is enveloped within the associated window; otherwise, it is coated in red.

\section{DATA AVAILABILITY}

The data that support the findings of this study are available from the corresponding author upon reasonable request.

\section{REFERENCES}

Arias-Castro, E., Kleissl, J., and Lave, M., “A Poisson model for anisotropic solar ramp rate correlations,” Sol. Energy 101, 192-202 (2014).

Bosch, J. L. and Kleissl, J., "Cloud motion vectors from a network of ground sensors in a solar power plant," Sol. Energy 95, 13-20 (2013).

Bosch, J. L., Zheng, Y., and Kleissl, J., "Deriving cloud velocity from an array of solar radiation measurements,” Sol. Energy 87, 196-203 (2013).

de la Parra, I., Marcos, J., García, M., and Marroyo, L., "Control strategies to use the minimum energy storage requirement for PV power ramp-rate control," Sol. Energy 111, 332-343 (2015).

de la Parra, I., Marcos, J., García, M., and Marroyo, L., "Dealing with the implementation of ramp-rate control strategies-Challenges and solutions to enable PV plants with energy storage systems to operate correctly," Sol. Energy 169, 242-248 (2018).

Fung, V., Bosch, J., Roberts, S., and Kleissl, J., "Cloud shadow speed sensor," Atmos. Meas. Tech. 7(6), 1693-1700 (2014).

Gevorgian, V. and Booth, S., "Review of PREPA technical requirements for interconnecting wind and solar generation," Report No. NREL/TP-5D00-57089) (National Renewable Energy Laboratory (NREL), Golden, CO, USA, 2013).

Hoff, T. E. and Perez, R., "Quantifying PV power output variability," Sol. Energy 84(10), 1782-1793 (2010).

Ineichen, P. and Perez, R., "A new airmass independent formulation for the Linke turbidity coefficient," Sol. Energy 73(3), 151-157 (2002).

Kuhn, P., Nouri, B., Wilbert, S., Prahl, C., Kozonek, N., Schmidt, T., Yasser, Z., Ramirez, L., Zarzalejo, L., and Meyer, A., "Validation of an all-sky imagerbased nowcasting system for industrial PV plants," Prog. Photovoltaics 26, 608 (2018a).

Kuhn, P., Wirtz, M., Wilbert, S., Bosch, J. L., Wang, G., Ramirez, L., Pitz-Paal, R. et al., "Field validation and benchmarking of a cloud shadow speed sensor," Sol. Energy 173, 229-245 (2018b).
Kuszamaul, S., Ellis, A., Stein, J., and Johnson, L., "Lanai high-density irradiance sensor network for characterizing solar resource variability of MW-scale PV system," in 35th IEEE Photovoltaic Specialists Conference (PVSC) (IEEE, 2010), pp. 000283-000288.

Lappalainen, K., Wang, G. C., and Kleissl, J., "Estimation of the largest expected photovoltaic power ramp rates," Applied Energy 278, 115636 (2020).

Lave, M., Kleissl, J., and Arias-Castro, E., "High-frequency irradiance fluctuations and geographic smoothing," Sol. Energy 86(8), 2190-2199 (2012).

Lave, M., Kleissl, J., and Stein, J. S., "A wavelet-based variability model (WVM) for solar PV power plants," IEEE Trans. Sustainable Energy 4(2), 501-509 (2013).

Lave, M. and Kleissl, J., "Cloud speed impact on solar variability scaling-Application to the wavelet variability model," Sol. Energy 91, 11-21 (2013).

Lave, M., Reno, M. J., and Broderick, R. J., "Characterizing local high-frequency solar variability and its impact to distribution studies," Sol. Energy 118, 327337 (2015).

Lave, M. and Weekley, A., "Comparison of high-frequency solar irradiance: Ground measured vs. satellite-derived," in IEEE 43rd Photovoltaic Specialists Conference (PVSC) (IEEE, 2016), pp. 1101-1106.

Marcos, J., de la Parra, I., García, M., and Marroyo, L., "Control strategies to smooth short-term power fluctuations in large photovoltaic plants using battery storage systems," Energies 7(10), 6593-6619 (2014a).

Marcos, J., Storkël, O., Marroyo, L., Garcia, M., and Lorenzo, E., "Storage requirements for PV power ramp-rate control,” Sol. Energy 99, 28-35 (2014b).

Marcos, J., de la Parra, Í., García, M., and Marroyo, L., "Simulating the variability of dispersed large PV plants,” Prog. Photovoltaics 24(5), 680-691 (2016).

Marcos, J., Marroyo, L., Lorenzo, E., Alvira, D., and Izco, E., "From irradiance to output power fluctuations: The PV plant as a low pass filter," Prog. Photovoltaics 19(5), 505-510 (2011).

Nguyen, A., Velay, M., Schoene, J., Zheglov, V., Kurtz, B., Murray, K., Torre, B., and Kleissl, J., "High PV penetration impacts on five local distribution networks using high resolution solar resource assessment with sky imager and quasi-steady state distribution system simulations," Sol. Energy 132, 221-235 (2016).

Pelland, S., Galanis, G., and Kallos, G., "Solar and photovoltaic forecasting through post-processing of the global environmental multiscale numerical weather prediction model," Prog. Photovoltaics 21(3), 284-296 (2013).

Perez, R., Kivalov, S., Schlemmer, J., Hemker, K., Jr., Renné, D., and Hoff, T. E., "Validation of short and medium term operational solar radiation forecasts in the US," Sol. Energy 84(12), 2161-2172 (2010).

Perez, R., David, M., Hoff, T. E., Jamaly, M., Kivalov, S., Kleissl, J., Perez, M. et al., "Spatial and temporal variability of solar energy," Found. Trends Renewable Energy 1(1), 1-44 (2016).

Ru, Y., Kleissl, J., and Martinez, S., "Exact sizing of battery capacity for photovoltaic systems," Eur. J. Control 20(1), 24-37 (2014).

Saleh, M., Meek, L., Masoum, M. A., and Abshar, M., "Battery-less short-term smoothing of photovoltaic generation using sky camera," IEEE Trans. Ind. Inf. 14(2), 403-414 (2018).

Shivashankar, S., Mekhilef, S., Mokhlis, H., and Karimi, M., "Mitigating methods of power fluctuation of photovoltaic (PV) sources-A review," Renewable Sustainable Energy Rev. 59, 1170-1184 (2016).

Tonkoski, R., Lopes, L. A., and El-Fouly, T. H., "Coordinated active power curtailment of grid connected PV inverters for overvoltage prevention," IEEE Trans. Sustainable Energy 2(2), 139-147 (2011).

Van Haaren, R., Morjaria, M., and Fthenakis, V., “An energy storage algorithm for ramp rate control of utility scale PV (photovoltaics) plants," Energy 91, 894-902 (2015).

Wang, G. C., Bosch, J. L., Kurtz, B., de la Parra, Í., Wu, E., and Kleissl, J., "Worst expected ramp rates from cloud speed measurements," in IEEE 46th Photovoltaic Specialists Conference (PVSC) (IEEE, 2019), pp. 2281-2286.

Wang, G., Kurtz, B., and Kleissl, J., "Cloud base height from sky imager and cloud speed sensor,” Sol. Energy 131, 208-221 (2016). 